\title{
Cardiac Prevention and Rehabilitation "3.0": From acute to chronic phase. Position Paper of the Italian Association for Cardiovascular Prevention and Rehabilitation (GICR-IACPR)
}

Roberto F.E. Pedretti', Francesco Fattirolli2, Raffaele Griffo3, Marco Ambrosetti', Elisabetta Angelino4, Silvia Brazzo5, Ugo Corrà 6 , Nicolò Dasseni7, Pompilio Faggiano7, Giuseppe Favretto8, Oreste Febo9, Marina Ferrari10, Francesco Giallauria11, Cesare Greco'12, Manuela lannucci13, Maria Teresa La Rovere ${ }^{10}$, Mario Mallardo'14, Antonio Mazza1, Massimo Piepoli15, Carmine Riccio'16, Simonetta Scalvini17, Luigi Tavazzi18, Pier Luigi Temporelli6, Gian Francesco Mureddu12

1 U.O. Cardiologia Riabilitativa, Dipartimento di Cardioangiologia Riabilitativa, Istituti Clinici Scientifici Maugeri, IRCCS di Pavia

2 S.O.D. Riabilitazione Cardiologica, Dipartimento di Medicina Sperimentale e Clinica, Università di Firenze, Azienda Ospedaliera Universitaria di Careggi, Firenze

3 Centro Studi per la Ricerca Clinica e la Formazione GICR-IACPR, Genova

4 U.O. Psicologia, Istituti Clinici Scientifici Maugeri, Presidio Sanitario di Torino, Torino

5 Ambulatorio di Dietetica e Nutrizione Clinica, Istituti Clinici Scientifici Maugeri, IRCCS di Pavia

6 U.O. Cardiologia Riabilitativa, Dipartimento di Cardioangiologia Riabilitativa, Istituti Clinici Scientifici Maugeri, IRCCS di Veruno (NO)

7 U.O. Cardiologia, A.O. Spedali Civili, Brescia

8 U.O. di Cardiologia Riabilitativa e Preventiva, Ospedale Riabilitativo di Alta Specializzazione,

Motta di Livenza (TV)

9 U.O. Cardiologia Riabilitativa, Ospedale Maggiore AAST-Crema, Presidio di Rivolta d'Adda (CR)

10 U.O. Cardiologia Riabilitativa, Dipartimento di Cardioangiologia Riabilitativa, Istituti Clinici Scientifici Maugeri, IRCCS di Montescano (PV)

11 U.O.C. di Medicina Interna ad Indirizzo Metabolico e Riabilitativo, AOU "Federico II", Napoli

12 U.O.C. Cardiologia e Riabilitazione Cardiologica, A.O. San Giovanni-Addolorata, Roma

13 Cardiac Prevention and Rehabilitation ONLUS Cardiosalus, Roma

14 U.O.C. Cardiologia e Riabilitazione Cardiologica, Ospedale San Gennaro, Napoli

15 Unità per lo Scompenso Cardiaco, Ospedale Polichirurgico, Ospedale Guglielmo da Saliceto, Piacenza

16 U.O. Cardiologia e Riabilitazione Cardiologica, Azienda Ospedaliera S. Anna e S. Sebastiano, Caserta

17 U.O. Cardiologia Riabilitativa, Dipartimento di Cardioangiologia Riabilitativa, Istituti Clinici Scientifici Maugeri,

IRCCS di Lumezzane (BS)

18 Maria Cecilia Hospital, GVM Care \& Research, Cotignola (RA), Italy

Corresponding author: Dr. Roberto F.E. Pedretti, U.O. Cardiologia Riabilitativa, Dipartimento di Cardioangiologia Riabilitativa, Istituti Clinici Scientifici Maugeri IRCCS di Pavia, Via S. Maugeri 10, 27100 Pavia, Italy. E-mail: roberto.pedretti@icsmaugeri.it

The Italian version of this Position Paper is published on Giornale Italiano di Cardiologia 2018;19(Suppl.3):10.

Key words: Cardiac rehabilitation; Secondary prevention.

Conflict of interest: The authors declare that no conflict of interest exists.

Received for publication: 22 October 2018

Accepted for publication: 22 October 2018

(C) Copyright R.F.E. Pedretti et al., 2018

Tipografia PI-ME Editrice, Italy

Monaldi Archives for Chest Disease 2018; 88:1004

doi: 10.4081/monaldi.2018.1004

This article is distributed under the terms of the Creative Commons Attribution Noncommercial License (by-nc 4.0) which permits any noncommercial use, distribution, and reproduction in any medium, provided the original author(s) and source are credited.
Abstract

Cardiac rehabilitation (CR) is the subspecialty of clinical cardiology dedicated to the treatment of cardiac patients, early and in the long term after an acute event. The aim of CR is to improve both quality of life and prognosis through prognostic stratification, clinical stabilization and optimization of therapy (pharmacological and non), management of comorbidities, treatment of disability, as well as through the provision and reinforcement of secondary prevention interventions and maintenaince of adherence to treatment.

The mission of CR has changed over time. Once centered on the acute phase, aimed primarily at short-term survival, the healthcare of cardiac patients now increasingly involves the chronic phase where the challenge is to guarantee continuity and quality of care in the medium and long-term. The aim of the present position paper is to provide the state-of-the-art of CR in Italy, discussing its trengths and weaknesses as well as future perspectives. 


\section{Executive Summary}

Cardiac Prevention and Rehabilitation (CPR) is the subspecialty of clinical cardiology dedicated to the treatment of cardiac patients in both the post-acute and chronic phases. Its goal is to improve the quality of life and prognosis through continued prognostic stratification, clinical stabilization, optimization of pharmacological and non-pharmacological therapy, management of comorbidities, treatment of disability, as well as through interventions and reinforcement of secondary prevention and the maintenance of adherence to therapy.

The mission of CPR has changed over time. From being centered on the acute phase, the challenge now increasingly is to guarantee continuity and quality of care in the medium and long-term chronic phase. The purpose of this position paper is to analyze the current status of CPR in Italy, focusing on its strengths but also weaknesses, and future prospects.

\section{Indication for CPR}

CPR continues to be indicated after acute coronary syndrome (with class I recommendation, level of evidence A), after coronary and valvular heart surgery, in chronic ischemic heart disease (with class I recommendation, level of evidence B) and in post-acute and chronic decompensated heart failure (with class I recommendation, level of evidence B). Whatever the context, the core contents of CPR are always the same, i.e. an overall assessment centered on the needs and goals of the patient, an intervention of clinical stabilization and therapy optimization, prescription and implementation of physical activity, educational support regarding the risk factors related to lifestyle and socio-psychological distress, specific counseling (also aimed at promoting treatment adherence) and finally a periodic evaluation of outcome. There is no indication for CPR in patients who have a limited probability of functional recovery, such as patients with significant motor disabilities or a pre-existing cognitive deficit at the time of the acute event, or in patients with a short life expectancy.

The photograph of CPR in 2013 in Italy shows 221 dedicated structures, a number which has been continuously increasing for over 30 years, still nonhomogeneous as regards the regional distribution, but with an average of one facility per 270,000 inhabitants. The rehabilitation provided by this network appears, albeit to a lesser extent compared to previous surveys, still very skewed towards the prevalently inpatient regime, sometimes also with high organizational complexity. In fact, $11 \%$ of the beds are described as equipped for sub-intensive care. Despite a strictly rehabilitative and non-specific code of discipline (code 56), the structures are governed and managed for the most part by cardiologists. But this problem of the heterogeneity of the CPR offer is not peculiar to Italy but pertains to Europe as a whole. For this reason, the need has become urgent to identify a platform based on the minimum services provided by CPR, which can be applied to all the realities. An important goal is certainly to align ourselves with what already happens in other countries, such as the United States, where the referral of each single patient to a CPR program is considered as an indicator of quality of care in acute care hospitals and the lack of referral earns a point of demerit, with repercussions on the reimbursement to the structure.

\section{Patients' selection and care setting}

In 2011, the consensus document ANMCO/GICR-IACPR "Criteria for the selection of patients for referral to centers of cardiac rehabilitation" had already introduced, alongside the indication for cardiac rehabilitation, the concept of "priority" of access to a CPR facility. Currently the increased prevalence of chronic conditions characterized by multimorbidity and associated with frailty and disability requires that we go beyond the treatment paradigm centered on management of the single pathology and embrace the complexity of care imposed by multimorbidity: for these reasons modern CPR needs to further reexamine the groups of patients who have access and the services provided. To give some examples, the problem of frailty must become fully a part of CPR, the physical exercise component must be re-elaborated as a function of the new therapeutic options offered to an ever-increasing number of patients (e.g. with transcatheter aortic valve replacement, left ventricular assist device as destination therapy) and, finally, the concept of rehabilitation must be expanded to include to prehabilitation. In this context, the Units of Intensive Cardiac Rehabilitation, already envisaged in the Ministry of Health's Rehabilitation Plan of 2011 under the term "units for severe disabling cardiac diseases", may represent a useful answer to the care needs of the patients above. In addition to inpatient CPR, the second "pillar" of CPR consists of the outpatient setting, for which various models exist but always with the need to respect a series of structural, organizational and management criteria, including the availability of multidisciplinary skills, to ensure the completeness of the programs.

The minimum operational criteria for a complete evaluation of secondary cardiovascular prevention, in the outpatient setting, must include a database with a data storage system (in a network of CPR centers) and a system for reviewing and managing diagnostic imaging. Nursing staff must be trained and standardized in basic counseling and systematic data collection. It is necessary that the clinic be coordinated by a clinical cardiologist with expertise in prevention and rehabilitation, and experience in cardiovascular imaging diagnostics, as well as in risk factor management, antithrombotic, anticoagulant and anti-ischemic drug therapy, pharmacological interactions, strategies for achieving therapeutic goals, and patient adherence/persistence regarding therapy.

\section{The elderly and the multidisciplinary care}

CPR today must also take up the issue of the inclusion of elderly patients in rehabilitation programs and the relative barriers to their access, including lack of patient motivation, insufficient knowledge about the benefits on the part of doctors, the accessibility to the facilities, and lack of a geriatric culture applied to traditional cardiology to face major challenges (frailty, sarcopenia, physical deconditioning, cognitive and psychosocial aspects). It is necessary to identify the specific problems, and the expectations and preferences of the elderly cardiac patient, defining the general and specific goals to pursue and using tools to detect these needs. As a general rule, one should use specific methods for these patients, starting from a "multidomain" assessment, followed by flexible and individually targeted interventions.

The multidisciplinary rehabilitation team (cardiologist, nurse, physiotherapist, dietician, psychologist) is by now a consolidated practice in CPR. But managing advanced age and multimorbidity calls for an interdisciplinary intervention involving consultations, also via functional connection, with the social worker and other specialists such as the pharmacist (for a second level opinion on the problem of drug interactions), cardiac surgeon, internist, diabetologist, neurologist, physiatrist, pulmonologist, nephrologist and infectivologist. In an era in which complex decisions are no longer taken individually but in a shared and multidisciplinary manner, often in well-defined functional groups such as the Heart Team, it is evident that also the rehabilitative cardiologist must be a full part of the decision making.

With a view to the appropriate allocation of resources and enhancement of the professional role of each professional figure, GICR-IACPR recently published the Minimal Care paths, i.e. modes of services provided and essential contents of the work performed by each professional figure. In elaborating the Minimal Care paths, the following dimensions were considered: the clinical-care complexity of the patient, the specific complexity for each professional area, the care setting and the duration of the program.

The achievement of an adequate clinical competence by all members of the interdisciplinary team in CPR settings is essential to provide an effective, safe and efficient intervention. As has already happened in other cardiological contexts, it would be advisable to draw up a true core curriculum for the health worker in CPR, reflecting the core components of CPR and adapted in relation to the different professional roles.

It is also very important that Patients' Associations increasingly become spokespersons of the importance of CPR, access to which too often is still not assured for many cardiac patients.

In a broader cultural context, CPR must also move in the direction of Digital Health, Precision Health and the use of Big Data, innovative components of medicine linked together by intra- and interconnections. CPR needs to project an image of itself as a medical area that is open, well-informed, sufficiently technologized to conduct etiopathogenetic investigations, able to accompany patients (who will play an ever-increasing part in determining the social role of medicine) with a participatory and highly professional approach, and which offers a service that is continuously available over time, associated with the confidence to obtain what medicine can give.

\section{Rate of referral to Cardiac Prevention and Rehabilitation}

Practically speaking, in recent years some Italian regions have drawn up regulations for a reorganization of the rehabilitation sector, sometimes even to the 
point of drafting of diagnostic-therapeutic care pathways thanks to the collaboration between rehabilitation cardiologists and the institutional exponents, the scope being to make the cardiac rehabilitation intervention as correct and appropriate as possible. It is therefore desirable that also in the Regions in which this has not yet been done, it will be possible to quickly set up ad hoc Technical Tables for an ever-increasing penetration of CPR.

However, a persistent critical point remains, i.e. that the current rates of referral to structured CPR programs by other health professionals active in the field of acute or community healthcare - particularly after the acute cardiac event but also in the chronic phase - are still unsatisfactory and impact unfavorably on the treatment of individual patients and on the prevention and control of cardiovascular diseases at the population level. Among the actions recognized as priorities to increase the rate of referral to CPR one can currently identify: 1) the need for a finer-grained analysis of the phenomenon within the field of CPR, with an adequate examination of the intrinsic dynamics of the stakeholders; 2) the need to achieve a process of professional and scientific accreditation of the network of Italian CPR facilities; 3 ) the need for adequate structuring and communication of the technical-logistical and operational characteristics of the individual CPR facilities (the so-called "Rehabilitation Facility Plan"), and a greater familiarization with it on the part of the providers referring the patients; and finally 4) the need for greater use of Patients' Associations to convey the message that CPR is a real "right of treatment".

Another difficult challenge which CPR is called to face is that of adherence to medical treatment, the key to success in almost all chronic conditions especially in cardiovascular diseases: the problem is more current than ever and is at the center of a lively scientific discussion.

Concerning innovation and research, CPR cannot fail to continue its effort to produce sound scientific evidence on the effectiveness of rehabilitation, in order to promote a greater interest in CPR especially on the part of acute-care cardiologists. This research effort must nevertheless be conducted with a more "modern" approach, i.e. not separating the fundamental constituents of "prevention" and "rehabilitation" and, above all, bearing in mind the technological innovations of the last few years as regards the availability of e-Health technologies and the harmonization between diagnostic-therapeutic care paths, identification/qualification of rehabilitation needs through the ICF classification (International Classification of Functioning), and the compilation of the individual rehabilitation program through digital applications.

\section{Future prospects}

From these issues outlined above, the opportunity now arises to talk about "CPR 3.0", which represents the latest development in the evolution from, first, an exclusively exercise-centric CPR ("CPR 1.0") and, then, multidisciplinary CPR ("CPR 2.0"), which already incorporated the activities of prognostic stratification, clinical stabilization and therapy optimization. In the present historical phase, characterized by an epidemiological evolution which has led to a dramatic rise in the elderly population with an equally important growth of chronic diseases, CPR sees the need to conduct a critical review and undergo a process of adaptation to this new reality.

\section{Introduction}

Cardiac Prevention and Rehabilitation (CPR) is the subspecialty of clinical cardiology to which cardiac patients should be referred after the acute cardiovascular event. It represents the logical place of care for the so-called post-acute (in reality, still acute) and chronic patients, and the physical "place" (in hospital or clinic) in which individual interventions of secondary cardiovascular prevention and adherence to medium and long-term therapy are planned and carried out. The goals of CPR are clinical stabilization and control of symptoms, reduction of the global cardiovascular risk, achievement of greater adherence to drug therapy, and implementation of a better lifestyle in order to achieve a better quality of life, better social integration and better prognosis [1,2]. The above goals are achieved through a multidisciplinary team coordinated by a cardiologist and composed of a nurse, physiotherapist, occupational therapist, dietician and psychologist, who can define a multidisciplinary intervention consisting of the following main components:

- Prognostic stratification and clinical stabilization,

- Counseling on physical activity,

- Prescription of a personalized physical training program,

- Counseling on diet and nutrition,

- Body weight management,

- Management of dyslipidemia,

- Blood pressure control and management,

- Smoking cessation,

- Psychological and social intervention.

The CPR develops and organizes its activities in three different phases [2]:

- Phase I: this refers to a very early intervention implemented in the most acute phase of a clinical event, usually in an acute hospital ward, and it includes early mobilization and prevention of complications related to immobilization;

- Phase II: refers to interventions implemented in patients admitted to CPR structures following a recent acute event with the following objectives: clinical stabilization, risk/prognostic stratification and promotion of long-term secondary prevention. This phase can be implemented in an inpatient or outpatient facility;

- Phase III: refers to a long-term outpatient CPR intervention aimed at providing a persistent prevention and rehabilitation intervention over time.

The role of secondary cardiovascular prevention has grown progressively in importance due to the significant changes in the clinical epidemiology of cardiovascular diseases that have taken place in the last two decades. Candidates for a CPR ${ }^{2}$ program can be patients with the following conditions:

- ischemic heart disease with particular regard to acute coronary syndrome,

- recent cardiac surgery and/or cardiac interventional procedure, both coronary and valvular,

- heart failure,

- heart transplantation,

- wearers of mechanical devices to assist circulation,

- wearers of cardiac electrical devices (permanent electrostimulation, implantable defibrillator, cardiac resynchronization),

- diabetes mellitus and metabolic syndrome,

- chronic obliterative arteriopathy of the lower limbs.

There is no indication for CPR in patients who have a limited probability of functional recovery, such as those with significant motor disabilities or pre-existing cognitive deficit at the time of the acute event, or in patients with a short life expectancy.

The above-mentioned diseases have a very significant epidemiological impact. Regarding just acute coronary syndrome and heart failure, the annual incidence of ST-segment elevation myocardial infarction in some European countries is between $43-144 / 100,000$ [3]. In the REGICOR registry, the annual incidence of myocardial infarction was $274.5 / 100000$ in men and 69.7/100 000 in women [4]. Although ischemic heart disease occurs on average 7-10 years later in women than in men, myocardial infarction remains one of the leading causes of death in females, and after 75 years of age women represent the majority of patients with acute coronary syndrome [3]. With regard to stable ischemic heart disease, the prevalence of angina in population studies increases with age in both sexes, and is between $5-7 \%$ in women aged $45-64$ years and $10-12 \%$ in women aged $65-84$ years; in men the prevalence of angina is $4-7 \%$ in the $45-64$ year age-group and $12-14 \%$ in the $65-84$ year age-group [5]. Regarding heart failure, the prevalence of heart failure is $1-2 \%$ of the adult population in developed countries, rising to $\geq 10 \%$ in people aged $>70$ years [6]. 
Management of heart disease is now a major problem especially in the post-acute and chronic phases, previously considered stable. Post-hospital morbidity and mortality have increased, particularly in patients with a high residual risk associated with the index hospitalization or with a high burden of residual cardiovascular risk [7]. Epidemiological, clinical and interventional studies confirm not only the importance of the period immediately following an acute event but open up the prospect of the need for long-term secondary prevention programs and care continuity models. For example, in Italy, in the clinical context of acute coronary syndrome, using administrative big data of hospital discharge forms, and defining as "high thrombotic-ischemic risk" patients discharged with at least one of the following: previous myocardial infarction or ischemic stroke, diabetes, peripheral vascular disease and renal dysfunction, high thrombotic risk predicted an increase of adverse events compared to the unexposed population, which increased during the first 18 months and remained stable for at least the following 5 years [8]. This observation confirms not only the importance of targeting intensive prevention therapeutic strategies in the period immediately following the acute event, but points to the need for long-term secondary prevention programs in subgroups of high-risk patients. In these patients, the secondary prevention program must be one of high intensity. Alongside coronary heart disease, one should also note the increasing incidence of diseases of highthromboembolic risk, often identified in the form of silent cerebral ischemic disease by imaging techniques. Heart failure and atrial fibrillation are largely incident diseases, closely correlated with each other and with the ischemic etiology, potentially able to determine recurrent events and severe disability and a greater risk of reduced treatment adherence both due to the coexistence of multiple comorbidities and to the complexity of the pharmacological strategies that they often demand. Valvular diseases are also in progressive and significant increase, as well as the possibility of their correction thanks to the technological development of interventional cardiology which now flanks cardiac surgery in providing solutions to clinical cardiologists and their patients.

It is therefore essential that patients at high risk of recurrent events be correctly identified, initiated on a strategy of intensive secondary prevention and, alongisde their often complex diagnostic-instrumental and pharmacological intervention (combinations of hypolipidemic drugs, double anti-aggregation, association between antiplatelet agents and anticoagulants with variable doses), be offered the opportunity to participate in a cardiac rehabilitation (CR) program, in continuity of care. CR is the best vehicle for secondary prevention interventions, which can lead to an increase in adherence to evidence-based therapies [9] and favorably condition the prognosis [10,11]. For these reasons, it is universally indicated with a high class of evidence by scientific guidelines and considered as an indicator of quality of care [1]. Nevertheless, to date, data from EUROASPIRE studies, as also data from observational studies carried out in North America and other countries, indicate that there is an insufficient control of risk factors in secondary prevention, largely due to the insufficient referral or non-referral of high-risk patients to rehabilitation programs.

The mission of CPR has therefore changed over time. From the acute intervention, aimed above all at short-term survival, we have moved on to the challenge of ensuring continuity and quality of care in the medium and long term. The so-called "post-acute" phase is frequently no longer characterized by a condition of clinical stability, and in this phase a great degree of pharmacological complexity is required to manage the complex therapeutic associations in patients who are often elderly and with multiple co-morbidities (renal failure and respiratory diseases, especially). The management of advanced age and multimorbidity requires the availability, also via functional connection, of an interdisciplinary intervention enabling consultation with the social worker, and other specialists such as the pharmacist (for a second level opinion on the problem of drug interactions), cardiac surgeon, internist, diabetologist, neurologist, physiatrist, pulmonologist, nephrologist and infectiologist [2].

This all corresponds in practice to an individually-tailored medicine, directed to the individual patient, and it requires adequate clinical skills, operational tools and care management organization. Therefore, in an era in which complex decisions are no longer taken on an individual level but in a shared and multidisciplinary manner, often in welldefined functional groups such as the Heart Team, it is evident that the rehabilitative cardiologist too must fully participate in this decisional process, in that this professional figure is able to provide an indispensable contribution in terms of clinical competence, proposal of care pathways and assistential capacity in synergy with that of the acutephase cardiology and other medical specialties that participate in the care path of the patient at high risk.

The purpose of this position paper is to perform an analysis of the current state of the art of CPR in Italy, focusing on the strengths but also on the weaknesses and future prospects. The use of a software number in the title of this position paper is not accidental - it serves to highlight our current position in a long path that began in the distant past. Over the decades we have witnessed a progressive and continuous evolution in our discipline, which began as an almost exclusively "exercise-centric" CPR (version "1.0"), to then become "multidisciplinary" CPR (version "2.0"). In the present historical phase characterized by an epidemiological evolution that has led (and will continue to lead) to a marked increase in the elderly population with an equally important growth of chronic diseases, it is important that CPR also carry out a critical review and undergo a process of adaptation to the new reality. Hence the need for a version " 3.0 ".

\section{BACKGROUND AND CURRENT SITUATION}

\section{Mission and expected outcomes of Cardiac Prevention and Rehabilitation}

Since many years, CPR, based on the evidence of its very favorable and cost-effective clinical-prognostic impact, is considered by all national and international guidelines and promoted by both Scientific Societies and independent regulatory authorities as an integral part of the care program for patients with cardiovascular diseases.

CPR has maintained in the guidelines, in an era marked by extraordinary advances in pharmacological therapies, particularly those of reperfusion [12], a class I indication with level of evidence A in patients after acute coronary syndrome (both with and without ST-segment elevation), in patients after surgical revascularization and in patients with chronic ischemic heart disease (class I recommendation, level of evidence B), always demonstrating a significant reduction in total mortality, cardiovascular mortality and rehospitalizations, with a marked improvement in quality of life [13., regardless of patients' age or sex or the organizational models. Also in post-acute and chronic heart failure (NYHA class II-III, with both reduced and preserved ejection fraction), the guidelines report a class I recommendation, with level of evidence $B$, based on the results of trials that have documented increased exercise tolerance and quality of life with a significant reduction in rehospitalizations and, in relation to the most severe patients, also significantly reduced mortality [6,14]. Even in patients with recent valvular heart surgery, there is an important indication for a CPR intervention early after surgery [15]. On the other hand, this long success story is not surprising, confirming that CPR is the most effective and efficient way of implementing secondary prevention in post-acute and chronic patients [2]. 
CPR has also proven to be a real laboratory of innovative research, intervention tools, evaluation methods, and therapies, similarly to the intensive care units for patients in the acute phase. It is in the context of CR, both inpatient and outpatient, that many specific skills have been created and developed, which are now part of the cardiology community as a whole. Skills in functional evaluation (from the 6-min walk test to the cardiopulmonary exercise test), in risk stratification for arrhythmic events and acute cardiac decompensation, in the study of the autonomic cardiovascular nervous system and in the implementation of innovative secondary prevention therapies. Strategies of non-pharmacological intervention have also been developed and defined, from a "scientifically appropriate" prescription of physical training to the evaluation (and consequent interventions) of therapeutic adherence to both medications and lifestyle, at a time when the latter problem was still not perceived either by the cardiology community or by the regulatory authorities [9].

The current, modern CR has kept its operational and cultural specificity intact over the years, continuing to present itself as an active and dynamic multifactorial care process based on a multi- and interdisciplinary team. The multi-interdisciplinary approach is a further extraordinary strength of CR. It aims, in post-acute or chronic patients, to promote the achievement, not only of the important goals mentioned above, but also of intermediate goals such as clinical stability, improvement of functional capacity, the reduction of symptoms and the overall risk profile (all of which are extremely important from the point of view of both the patient and caregiver).

However, if on the one hand the ability to stratify cardiovascular risk has been increasingly refined, the same cannot be said for the ability to stratify prevention interventions, i.e. the ability to choose and adapt to the specific needs of the individual patient the tools that should - or can - be used. In this regard, the information provided by guidelines and statements does not seem sufficient to define objectively the priorities for intervention. One of the challenges of CPR is to evaluate, define and select interventions based on the characteristics and needs of the individual patient in everyday clinical practice. This competence appears even more urgent in an era of aging population and greater complexity of the patients affected by cardiovascular diseases. In fact, the complexity is now related not simply to the underlying cardiovascular pathology (and its distance from the index event) or to the cardiovascular risk profile but rather to other specific elements. Factors such as comorbidities, the risk/presence of cognitive or motor disabilities, psychological and mood disturbances, the socio-economic context, or problems related to nutrition, to the predisposition to adherence, to empowerment, and to patients' or caregivers' expectations/aspirations.

Customizing the interventions to the individual patient's needs, and identifying together the priorities and the sustainability of the goals is today the true daily challenge for health professionals in CPR, in whatever organizational context they are operating [16].
Whatever the setting of the CPR program, it is necessary to define an individual program that includes the fundamental components already enunciated in the foregoing introduction.

It is therefore essential to develop greater skill in customizing the assessment approach and intervention modalities to the needs of the individual patient. Consequently, it is of great importance for the future of CPR to develop training and refresher programs - for cardiologists and other professionals - aimed at maintaining or renewing skills in developing CPR programs tailored to the needs of the individual and not based exclusively on cardiovascular risk.

In a global context of scant accessibility to the CPR programs and of often only partial results from the standard cardiological intervention, it is time to update our armamentarium to a suitable standard for rehabilitative and preventive care, articulating the levels of care intensity in relation to the nature of the needs and to sustainability. From this stems the need to redefine the goals and methodologies of CPR, starting from an evaluation of the different "areas" in which to plan interventions based on flexible programs (Table 1).

In the light of this careful analysis of the patient, it will be easier to choose the right skills and setting required, widening the options beyond the inpatient setting to include also outpatient, extra-hospital and community care. This attitude is necessary in order to overcome the current "bottleneck" in implementing a CPR intervention that is effective for all the patients indicated for it.

\section{Cardiac Prevention and Rehabilitation facilities in Italy}

At the last survey in 2013 [17], 221 CPR facilities were registered in Italy ( 1 for every 270,000 inhabitants) representing an increase of over $20 \%$ (even higher in the south of the country) with respect to the previous five years. The facilities are largely located in hospital structures (around $60 \%$ public and $40 \%$ private) and there are wide differences in distribution throughout the country. Of these, 130 offer inpatient CPR services with 3527 beds ( $+27 \%$ compared to 2008 ), of which 408 equipped for sub-intensive therapy, and 102 offer outpatient CPR services (61 are exclusively outpatient centers offering this as the only mode of treatment). The experiences of facilities that use home rehabilitation are completely anecdotal.

Regarding classification of the facilities, $65 \%$ are classified in the Ministry of Health health information system with the disciplinary code 56 (functional recovery and rehabilitation), while $27 \%$ are instead classified with the acute cardiology code. Seventy-seven percent of the complex structures are directed by a cardiologist, $91 \%$ are simple. of the 111 structures inserted within a hospital department, $39 \%$ are part of a Cardiovascular Department.

Based on the discharges made in one week of sampling, the treatment potential of the CPR network is about 70,000 patients/year in hospital/day-hospital regime and about $30,000 /$ year in the outpatient regime. The total offer represents, as in all other advanced countries,

Table 1. Methodology of Cardiac Prevention and Rehabilitation: Areas to prioritize.

- Patient problems and expectations

- Definition of specific target priorities to be pursued:

- For functional recovery

- For secondary prevention

- For the prevention/correction of patient risks

- Assessments with appropriate tools

- Customization of programs

- Operational modes of implementation of programs

- Involvement and enhancement of patient preferences 
not more than $30-35 \%$ of the patients for whom CPR would be indicated.

The survey also revealed large differences in staff numbers per operating unit: the total number obviously increased in absolute terms due to the increase in the number of operating structures, but there was a decrease both in terms of number and time commitment per structure compared to the previous census conducted in 2008. For example, the number of cardiologists involved in structures providing CPR services was 772 in 2013, of which more than $40 \%$ dedicated only part-time to these activities. What also emerged very clearly was the severe shortage of psychologists and dieticians, even those dedicated only part-time.

This is reflected in some centers in a systematic incompleteness of the assessment and intervention program or, in other cases, in the substitution by other professional figures with non-verified expertise. Even this aspect should not be surprising: the Italian data are unfortunately in line with what is found in other Western countries [18], where the staff members consist substantially of three professional figures: cardiologist, physiotherapist and nurse. As a result of these organizational and cultural deficiencies the expected health outcomes may not be assured in all patients.

Finally, no quantitative or qualitative data exist to our knowledge concerning the numerous Secondary Prevention Clinics functionally correlated to Cardiac Intensive Care Units/Acute Cardiology.

These findings prompt several reflections:

1. It should be stressed that the breakdown described above refers to the year 2013. Since then, it is highly reasonable to assume that the situation may have evolved unfavorably, given the progressive reduction of available resources. It is a common experience to witness the reorganization processes of health structures, not infrequently involving cardiological activities including rehabilitation.

2. The fact that the rehabilitation facilities are directed in only about $70 \%$ of cases by a cardiologist represents an evident problem, first of all for patients. This aspect, besides being against common sense and patients' interest, is in clear contrast with the international guidelines on the subject [2].

3. It has always been stressed that the referral to structured secondary prevention or rehabilitation programs is unsatisfactory [19], but, paradoxically, if this were to increase, it would not be able to be satisfied by the current network in Italy, capable of absorbing just over one-third of patients with an elective indication. To adequately respond (in theory) to this inderogable health need (as CPR demonstrates to be, with its results) the main road is certainly to increase the supply. Not by adding new resources (unrealistic even to imagine in the present socio-economic climate), but rather by redirecting and reorganizing the activity of the existing structures that are no longer adequate to meet patients' needs either at outpatient or inpatient level. This reorganization means not simply a change of "label", but reorienting these services so that they can effectively ensure the minimal characteristics of a CR intervention in daily practice. CR for its part is already doing all it can within its current possibilities: i.e. verifying and supporting the development of its network also from the point of view of quality (program components, process and outcome indicators, standards); discussing openly with the whole cardiology community the indications for and appropriateness of CPR interventions; increasing the attention of cardiologists, cardiac surgeons, providers, institutions, patients' associations and public opinion on the growing need generated by the post-acute and chronic phases of cardiovascular diseases.

4. It is very important that during their specialization training, young cardiologists receive adequate training on secondary prevention and rehabilitation.
5. It is necessary to identify low-intensity models for the delivery of CPR programs for less complex patients utilizing/creating community resources. But this presupposes, upstream, a great capacity (to be developed and disseminated) in the stratification of needs and interventions, identifying what are "the right things to do, at the right time and in the right patient" (as can be seen in the Minimal Care [20] document). And, downstream, structures that are "light" but professionally competent and able to ensure effective intervention, avoiding the risk of a reductive vision that would identify CPR with simplified and non-characterized models, emphasizing instead the exclusive "core" contents and competences.

6. That the survey contains only anecdotal evidence on the experience of home rehabilitation requires reflection. As will be seen in the following sections, proposing innovative models and tools for care and control in this era of low-cost ad-hoc home-internet appbased devices, that make it possible to assess remotely the quality and effectiveness of the program, is a necessity and an area in which CPR will undoubtedly have to commit itself and operate.

\section{Cardiac Prevention and Rehabilitation outside Italy}

Research in the field of cardiovascular prevention has promoted the evolution of programs of CR, once focused only on functional recovery after a cardiovascular event, into multi-disciplinary centers of secondary prevention [21] adopting the aims of CPR to reduce risk and cardiovascular events and to promote healthy behaviors through an appropriate lifestyle [1].

The World Health Organization (WHO) and the Word Heart Federation (WHF) have resolved to reduce the risk of death from noncommunicable diseases by $25 \%$ in 2025 [22], through acting on the cardiovascular disease risk factors. The WHO and WHF intentions are summarized in Figure 1. In the world, about 17 million people die annually from cardiovascular disease and from 24 to 51 million suffer from a non-fatal form; it is estimated that in 2030 , cardiovascular disease will be the main cause of deaths/year [22]. Therefore, the WHF has formulated a document underlining the need to act in a coordinated and global manner and has designed a path to overcome the barriers at the level of national health systems, health professionals and patients to promote the adoption of the guidelines for cardiovascular disease prevention (Table 2).

CPR is a fundamental step in the field of secondary prevention [9]: it is a consolidated, all-encompassing, flexible procedure open to hospitalization and/or outpatient models for care, support and monitoring. In Europe, local and national prototypes affect the diffusion and application of CPR. In 2010, the results of the European Cardiac Rehabilitation Inventory Survey (ECRIS), promoted by the European Association for Cardiovascular Prevention and Rehabilitation [23], were published. The survey involved 28 European countries, about $72 \%$ of the members of the European Cardiology Society (ESC), about 10,000 acute care hospitals and about 45,000 cardiologists. Although the results are conditioned by the number of respondents (72\%), ECRIS has documented the national scientific references, resources, services (Phases I, II and III) and professional figures involved across European countries. In fact, ECRIS has underlined the extreme European variability, reflecting the economic resources available and the local scientific (cultural) dispositions.

Sixty-one percent of European countries have a national organization to refer to and $86 \%$ are involved in Phase I CPR [23]: this figure is fragmented and incomplete since 50\% of European countries did not respond. The adherence to Phase I CPR is in contrast to the short stay in hospital for acute patients and to the chronic nature of cardiovascular disease. It would be appropriate to continue treatment and pre- 


\section{Specific aims of the World Health Organization to combat noncommunicable diseases (NCDs): Clobal target $25 \times 25$}

$\mathbf{2 5} \%$ reduction of mortality due to cardiovascular diseases, cancer, diabetes and respiratory diseases by 2025

\section{Specific strategies of the World Heart Federation for the secondary prevention of cardiovascular disease (CVD)}

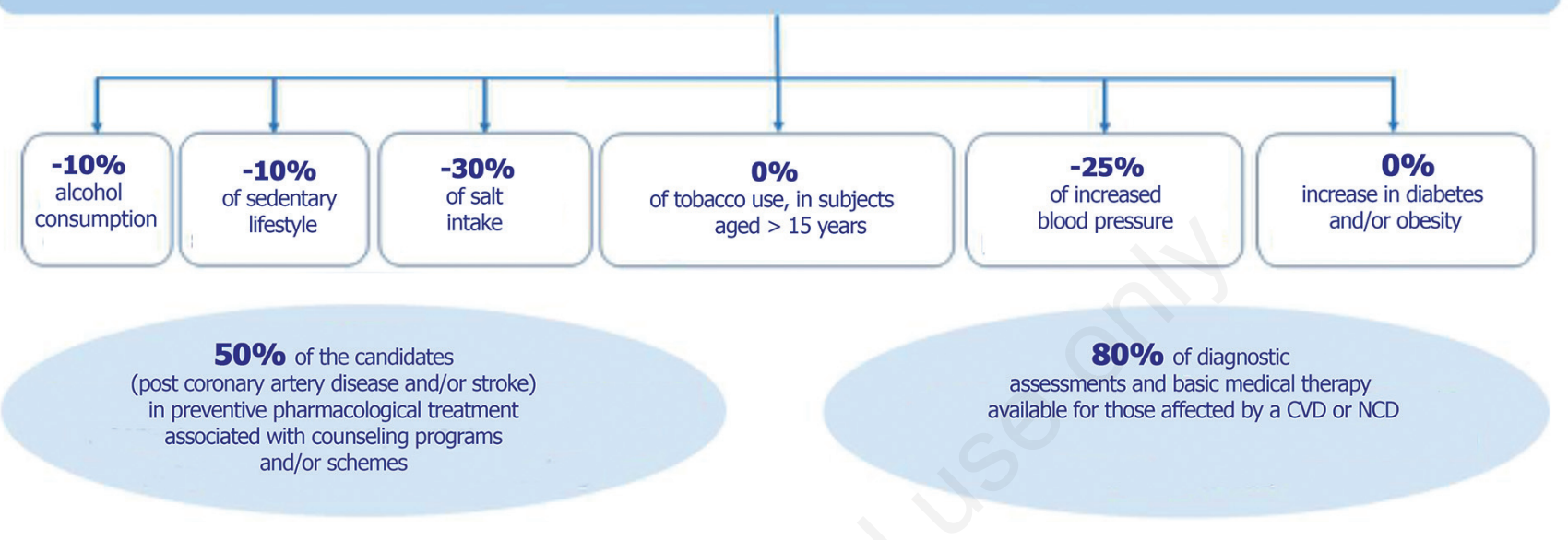

Figure 1. World Health Organization and World Heart Federation strategies for reducing the mortality risk due to noncommunicable diseases by 2025 .

Table 2. Suggestions/recommendations from the World Heart Federation.

\section{For national policymakers}

1. All countries should have a national program for CVDs providing for secondary prevention, with precise time targets and supported by funds-resources.

2. Monitor adherence to the guidelines for an adequate care, secondary prevention and rehabilitation.

3. Identify and combat any financial restraints that reduce the implementation of the guidelines with a consequent impact on the health services/clinical care provided to the patient.

4. Ensure equal access to health services (including those specific to CVD).

For organizations and societies responsible for disseminating and implementing the prevention guidelines

1. Prepare concise, clear guidelines that are appropriate to the national context. If multiple guidelines exist, the WHF suggests unifying them for better clarity and diffusion.

2. Provide the health professionals involved with effective training packages regarding the implementation of guidelines and support to better coordinate communication between them and patients with CVD.

3. Consider the (potential) help provided by technology.

4. Identify and combat any financial restraints that reduce the implementation of the guidelines with a consequent impact on primary and secondary prevention.

5. Explore and promote effective evidence-based strategies for dissemination and implementation of guidelines.

For health professionals

1. Improve coordination between primary and secondary care in order to have a holistic view of the patient and ensure that secondary prevention is part of the healthcare system.

2. Establish individual care for patients with CVD, with appropriate use of secondary prevention services.

\section{For patients}

1. Raise the level of secondary prevention of CVDs, of equal access for all to treatments and services, and of adherence to the implementation of the guidelines.

2. Identify and combat any financial restraints that reduce the implementation of the guidelines with a consequent clinical impact on the individual.

3. Clear and precise aspects (for patients) regarding prevention guidelines.

CVD, cardiovascular disease; WHF, World Heart Federation. 
vention with the services of CPR depending on the patient's severity, optimization of cardiovascular therapy and the residual risk profile [1].

According to the survey, phase II CPR existed in all European countries and in $64 \%$ of cases it was available for both inpatients and outpatients. The type of service varied and the duration, in most cases, was $6-12$ weeks [23]. The patients participating in phase II were not homogeneous as regards either the numbers or the cardiac pathology. For example, about $3 \%$ of patients participated in phase II CPR in Spain vs. up to $90 \%$ in Lithuania. As regards the heart disease profile, those who participated were mostly patients following myocardial infarction (82\%) or myocardial revascularization (86\%): some centers received patients after coronary angioplasty without heart attack (36\%), while more than $50 \%$ of European countries received patients after valvular heart surgery or heart transplant [23].

About $89 \%$ of European countries offered a phase III form of CPR [23]. There were marked differences, however, in the duration of the programs; $31 \%$ proposed phase III CPR programs of short-duration (3 to 12 weeks), but many offered longer-term programs [20 to 52 weeks $(n=4) ; 1-2$ years $(n=3)$; life-long programs $(n=4)]$. Also with regard to the maintenance phase, the offer was varied and conditioned by local regulations.

In fact, Europe is a continent of different countries in terms of healthcare distribution: each country is subject to state-regional laws and regulations. Therefore, the diffusion and type of CPR proposed is heterogeneous. An efficient audit could provide a minimum proposal for CPR, characterized by "sample" models that can be applied to all European realities. This approach is currently missing.

The multiform reality of CPR in Europe contrasts with what is happening in the United States [24], where there is intensive work ongoing on key aspects of public health. A recent, fundamental, document of the American College of Cardiology and of the American Heart Association [25] discusses the clinical performance and the quality measures of cardiac rehabilitation and clearly establishes the various operational steps of a rehabilitative path. In addition to defining, based on the published evidence, the candidates for rehabilitation it specifies the tasks of the cardiologist in performing the activities and useful tasks to encourage the participation of each individual patient.

\section{MODULATING PATHS OF INTERVENTION BY THE GOALS AND INTENSITY: "STRATIFICATION" OF THE REHABILITATION OFFER}

\section{Access priority for inpatient rehabilitation: how to apply the recommendations of the ANMCO/GICR-IACPR 2011 Consensus Document}

In 2011, the ANMCO/GICR-IACPR Consensus document "Criteria for the selection of patients for referral to centers of rehabilitation cardiology" introduced, together with the indication for cardiac rehabilitation, the concept of "priority" of access to CR [26]. It defined the precise categories of patients for whom the CR intervention is not only useful but an indispensable priority, and for whom access to dedicated facilities must be ensured. To identify the categories which have an absolute indication for rehabilitation, the document referred to the risk level of patient groups, with the aim of ensuring that the rehabilitation benefits were first provided to those at higher clinical risk, in the belief that intervention on these patients was more effective in terms of outcome. The document also aimed to redefine the modes for performing CR activity since, with rehabilitation indicated as a priority for the minority of patients at higher risk in the different disease categories, the organizational potential for inpatient CR would logically be exceeded. It thus seemed necessary to reformulate the rehabilitation offer through a more limited use of inpatient CR, both in terms of patient selection and length of stay, and an increase of outpatient or also home-based CR for those at lower risk.

For patients suffering from acute coronary syndrome, the panel decided they had priority of access to an inpatient CR facility in the postacute phase, in the following cases of infarcted patients at high clinical risk:

- myocardial infarction with decompensated heart failure or with left ventricular dysfunction (ejection fraction $<40 \%$ );

- myocardial infarction with prolonged stay in acute care or with complications or comorbidities;

- myocardial infarction in people with extremely sedentary lifestyle or elderly.

The panel considered preferably outpatient rehabilitation as a priority for patients with high cardiovascular clinical risk in cases of:

- incomplete revascularization, diffuse or critical coronary disease, multiple risk factors, resistance to lifestyle changes, especially in young patients.

An outpatient rehabilitation program was considered a priority, especially where psychologist consultation is available, in patients with reactive anxious-depressive syndromes.

The document gave an indication, but not a priority, for rehabilitative treatment to patients at low clinical risk (about $70 \%$ of post-infarct patients) and those with a medium-low cardiovascular risk based on the risk cards. To these patients, correct information/education must be guaranteed concerning their illness and the lifestyle habits to follow.

For patients with heart failure, access priority to an inpatient CR facility was given for those:

- in prolonged unstable conditions (mainly in NYHA class III-IV), especially with the need for pharmacological therapies in the phase of titration or infusion therapies and/or requiring intensive rehabilitative treatment (intensive health education, physical training or reconditioning);

- undergoing evaluation for cardiac transplantation or verification of persistence of the indication.

For patients following cardiac surgery, the panel considered access to an inpatient CR facility as a priority for the following complicated or complex patients:

- patients at high risk of new cardiovascular events and/or clinical instability, defined by: the presence of heart failure (NYHA class $>$ II or ejection fraction $<35 \%$ ) or severe hyper-hypokinetic arrhythmias, or the need for infusion therapies or early recurrent ischemic events or other factors of instability;

- patients discharged late after a prolonged stay in intensive care or intensive respiratory/cardiac care;

- patients with event-related complications, such as cerebral stroke, transient ischemic attack, cognitive impairment, renal failure (de novo or aggravated or requiring replacement treatment), pulmonary embolism, resurgery, pleuropericardial effusions requiring evacuation therapy, systemic infections, complicated wounds or decubitus;

- patients with presence or exacerbations of a severe comorbidity (chronic obstructive pulmonary disease, chronic respiratory failure, diabetes, neurological outcomes), exacerbations of other internal comorbidities, cognitive deficits (Mini-Mental State Examination), dysautonomia or frailty, need for complex pharmacological treatments, including nutritional, and individual rehabilitation interventions;

- patients with logistic/environmental/socio-welfare difficulties (psycho-social-health problems) complicating or delaying the discharge due to the need for actions to make it feasible (contacts with social-health services, etc.). 
The document also underlined that, compatibly with the clinical conditions, the subsequent transition from inpatient to outpatient CR should in any case be promoted, reducing the average length of stay.

At a distance of 7 years, it is reasonable to reappraise these indications in the light of new scientific evidence that may have come to light in the meantime and changes introduced at a regulatory and institutional level.

As regards ischemic heart disease, the ANMCO/GISE/GICR-IACPR consensus document "The organization of care in the post-acute phase of coronary syndromes" is a more recent reference that validates and reinforces the indications of 2011 [27]. In particular, it stresses the importance of a correct approach to formulating the diagnosis of heart failure, and for identifying the patients at risk, using all the indicators. The document suggests the use of a check-list in the acute-care medical record that includes: maximum Killip class, echocardiographic ejection fraction, remodeling predictors, use of loop diuretics for dyspnea or pulmonary stasis of non-secondary origin and, finally, variation in brain natriuretic peptide levels. These parameters should be recorded during the hospital stay and included in the discharge letter to guide the subsequent care path. Patients with heart failure so identified should be referred on discharge to a protected inpatient CR pathway. National epidemiological data confirm that, besides a particularly negative 1-year prognosis with $>10 \%$ mortality, this category of patients has a high rate of readmissions ( $64 \%$ of all-cause readmissions at 1 year, of which $20 \%$ for heart failure) with a predictable marked increase in care costs.

For patients with heart failure, the 2009 HF-ACTION [28] data showing significantly reduced readmissions in patients who underwent CR programs were confirmed by a 2014 Cochrane meta-analysis based on 15 studies with 1328 participants, in which exercise in CR programs reduced both all-cause readmissions [ 15 trials, 1328 participants: relative risk (RR) 0.75; confidence interval (CI) 95\% 0.62-0.92] and heart failure-related readmissions (12 trials, 1036 participants: RR 0.61; 95\% CI 0.46-0.80) [14].

For patients' post-cardiac surgery, even in the absence of recent data, it seems reasonable to state that in this area the indications of the 2011 consensus document have become and are now easier to apply, in the light of current clinical practice.

Application of the document recommendations, however, is in relation not only to the growing body of scientific evidence, but also to organizational needs of the health system. Over and above the scientific aspects, the rationale in the current healthcare organization for the above-mentioned indications for inpatient CR is twofold:

- the use of an inpatient CR program after an acute-care stay in patients with myocardial infarction or with an episode of heart failure at high risk can, similarly to what happens for cardiac surgery, reduce the hospital stay in acute care, absorbing the more severe and/or complex patients, thus leading to a more efficient use of acute care and rendering it more available for the needs of emergency care;

- the use of inpatient CR programs can significantly reduce readmissions. The number of readmissions is rapidly becoming a parameter of quality of care now used in most Regions. As is known, this is by far the largest item of expenditure after a first admission in acute care, e.g. it represents $85 \%$ of the healthcare expenditure in the year following an acute coronary syndrome.

At this point it is reasonable to ask why, in the presence of two strong motivations - one administrative, the other pertaining to the "system" - and in the face of robust scientific evidence, the use of CR is still insufficient according to the consolidated indications. The answer to this question is at different levels.

The articulation of CR structures is strongly differentiated and often they are located outside the acute facilities and belong to different bodies. This makes it more difficult to evaluate the benefits that can be obtained in terms of saving resources through the use of an inpatient cardiac rehabilitation program. In addition, the monitoring of patient flows at the regional level is still incomplete.

We need to validate in the Italian reality what the literature tells us on these issues, both as regards reduced length of stay, lower rate of readmissions and improved prognosis. Such validation can serve as an instrument to use in dealing with regulatory bodies and local authorities.

The data presented at the last ESC Congress represent a first important step in this direction [29]. In fact, the report analyzes two groups of patients from the Lombardy Region database, discharged with a diagnosis of heart failure between 2005 and 2012. The first cohort of 100,841 patients was admitted only to acute hospital wards while the second group of 39,709 was admitted at least once also to a CR facility. After adjustment for the different covariates, mortality in the second group was $43 \%$ lower and readmissions were reduced by $31 \%$ in the CR group.

It is evident the importance of these data that confirm the effectiveness of rehabilitation treatment in Italy. It would be desirable to replicate this analysis also in the post-infarct field, where the potential effect of rehabilitation is even greater. Analyses of this type should be proposed to regional health bodies in that they are a condition for optimizing resources and improving results.

\section{Inpatient Cardiac Prevention and Rehabilitation: Ever-increasing clinical complexity}

The increase in life expectancy and the rapid and continuous evolution of cardiovascular medicine have substantially changed the epidemiological picture of cardiovascular diseases, with an increase in the prevalence of chronic conditions characterized by multimorbidity and associated with frailty and disability. In Italy, where more than one in 5 people are over 65 years, the "elderly" (75-84 years) number over 4.8 million, while the population of "older adults" ( $\geq 85$ years old) is almost 2 million. Over the past decade, the international cardiology community has examined the need for the cardiologist to have adequate cultural tools to cope with the emerging health and economic challenges resulting from these demographic changes. In Italy, as well as abroad, there is a strong process of cultural integration underway between Cardiology and Geriatrics that has led to the formal definition of Geriatric Cardiology. Its aim is to identify treatment processes that are patientcentered and adequate to meet the multiple needs of this complex population [30]. The need to go beyond the treatment paradigm centered on management of a single pathology and embrace the complexity imposed by multimorbidity is the subject of a recent document of the American College of Cardiology [31].

CPR - by virtue of its multifactorial care construct based on a multidisciplinary team that incorporates not only the medical but also the social, psychological and behavioral dimensions of the disease - is in a privileged position as regards the cultural approach to the clinical complexity of multimorbidity.

The high clinical risk stated in the joint-society consensus document ANMCO/GICR-IACPR [26] as a priority and indispensable criterion for inpatient CR care has progressively widened to embrace new conditions and new access groups. This creates a constant need to update the skills of the health professionals and the services performed to take charge of the new needs of increasing clinical complexity.

To give some examples, the need to evaluate and manage the problem of frailty, the need to reprocess contents and programs related to the exercise component in virtue of the new therapeutic options now offered to a broader range of individuals (from those after transcatheter aortic valve replacement to those receiving a ventricular assist device as destination therapy), the need to extend the concept of 
rehabilitation from "recovery downstream of an event" to the prehabilitation, i.e. the optimization of physical and psychological capacities in a stage preceeding the interventional procedure.

Frailty, defined as a biological syndrome that reflects a reduced physiological reserve and an increased vulnerability to stressful events, is becoming increasingly important in the context of cardiovascular disease [32]. Regardless of the diagnostic tool used, in various populations of patients with cardiovascular disease the presence of frailty has proven to be superior as a prognostic indicator to many other known risk indicators. In particular, in patients who underwent aortic valve replacement (both transcatheter and surgical), the presence of frailty was of primary importance in conditioning both the success of the intervention and the potential for recovery in the absence of permanent disability [33]. Unlike patients with an indication for aortic valve replacement in whom the presence of frailty is part of the geriatric syndrome typical of this patient group, in patients with heart failure the presence of frailty is not related to age or functional class and has a prevalence which varies according to the instrument used, but is overall around 50\%. Although there is still no consensus on what is the most suitable tool to assess frailty in the rehabilitative context, the assessment/measurement of frailty will have to increasingly become part of the clinical routine in the functional assessment of patients referred to CPR in order to plan their individualized treatment. The results of the pilot study REHAB-HF [34] demonstrate that an early intervention in elderly subjects with recent acute heart failure that was individually tailored on the basis of a measure of physical frailty, the Short Physical Performance Battery, was able to reduce frailty and all-cause hospitalizations in the following 6 months.

The specificity of the role of CPR - both inpatient and non - in the elderly and very elderly patient is dealt with extensively in a later paragraph.

The development and implementation of physical activity programs and nutritional interventions aimed at preventing, reducing or "treating" frailty is the challenge of the future for inpatient CPR, and resources need to be invested for clinical research in this area.

In patients with clinically unstable heart failure and at high risk of early rehospitalization, inpatient CPR is the ideal place to implement that multidisciplinary management to which the guidelines attribute a class I recommendation with level of evidence A. As data show from the national registry IN-HF Outcome [35] and the National Outcome Program (http://pne2017.agenas.it/.) reporting 30-day readmission rates of $6.2 \%$ and $15.3 \%$, respectively, inpatient CPR is particularly well suited to manage the problems that accompany the so-called "vulnerable phase" after an acute-care stay, i.e. to play the role of "transitional care". Among the many factors that determine patients after an acute event at risk of short-term readmission, a major one certainly is the persistence of hemodynamic congestion, despite the improvement of symptoms. Recent data from the administrative database of the Lombardy Region presented at the ESC Congress regarding more than 140,000 subjects with heart failure incident in the years 2005-2012, of whom $29 \%$ underwent CPR, showed that the transition to one of the rehabilitation cardiology facilities reduced mortality and readmission and favored the implementation of the recommended therapies [29]. It is important to note that admission to CPR only rarely followed the "incident" episode; it tended to be recommended only after several episodes of instability and readmissions to acute care (on average after $3.26 \pm 1.78$ hospitalizations). Concerning the clinical characteristics of the patients who underwent CPR, they were more frequently male, of a more advanced age in women and had a greater burden of comorbidities and interventional procedures $(\mathrm{p}<0.0001)$. Prescribing of the recommended therapies and frequency of follow-up visits were more frequent in patients who underwent CPR $(\mathrm{p}<0.0001)$. After adjustment for different covariates, the risk of all-cause death and readmission in patients undergoing CPR, was, respectively, 0.5768 (IC 0.5650-0.5888, $\mathrm{p}<0.0001$ ) and 0.7997 (IC 0.7758-0.8244, p<0.0001) [29].

Patients implanted with a left ventricular assist device, due to their high levels of deconditioning and functional impairment and multiple clinical and psychological needs, are natural candidates for an inpatient pathway of CPR. In a recent analysis of 1164 patients implanted with a ventricular assist device in 2014 in the United States, of whom $30 \%$ were referred to cardiac rehabilitation, participation in the rehabilitation program was associated - at 1-year follow-up - to a $23 \%$ lower risk of rehospitalization $(\mathrm{p}<0.001)$ and $47 \%$ lower mortality $(\mathrm{p}<0.01)$ [36]. The treatment of these patients requires precise and dedicated skills, while the methods for administering treatment and the physical exercise programs still need to be standardized. The development and diffusion of these skills are an area in which inpatient CPR needs to grow culturally and scientifically in the coming years [37].

The concept of rehabilitation must be extended to prehabilitation. Frailty in the surgical patient (in cardiac surgery, more than $10 \%$ of total interventions are in people aged $>80$ years) is associated with a 3 -fold increase in postoperative mortality and morbidity and is associated with major cardiovascular and cerebrovascular events. In preliminary studies, preoperative improvement of functional capacity, nutritional status and mental status was effective in improving postoperative outcome and recovery after surgery. It is important to underline that interventions of prehabilitation are not a specific prerogative of inpatient CPR, but can also be applied in less intensive contexts depending on the individual characteristics.

\section{Units for severe disabling cardiac diseases: Intensive Cardiac Rehabilitation Units}

Improved levels of care and new technologies have in recent years created a new patient population that we can define as "survivors of catastrophic pathological events". These patients often require prolonged weaning procedures from dependence on numerous devices, in particular mechanical ventilation and the need for vasoactive therapy (amines, sodium nitroprusside, diuretics, etc.) in continuous or intermittent infusion.

In cardiology, the stereotype of the above clinical condition is the patient with acute heart failure, and the "chronically unstable" patient who is refractory to pharmacological and non-pharmacological drugs and classifiable as suffering from advanced heart failure (stage D of the American Heart Association) [38]. Also patients with recent acute coronary syndrome or with complications post-cardiac surgery requiring high intensity care can be considered "survivors of catastrophic pathological events".

In the Ministry of Health 'Plan for Rehabilitation' of 2011, in the section dedicated to hospitalization and care settings, among emerging needs in rehabilitation, the document deals with the establishment of spinal units and units for severe cardiorespiratory disabilities, characterized by dedicated multidisciplinary teams and logistic and functional structures with specific advanced technologies [39].

The 2011 ministerial document describes the Unit for Severe Cardiac Diseases as "intended for the care of patients in a state of clinical instability due to a very recent acute cardiovascular event, the persistence of complex post-acute or surgical problems, or heart failure refractory to conventional therapy, but with potential for clinical-functional recovery. The interventions for the patients of the Unit are focused on definitive clinical stabilization, the restoration of autonomy of the basic vital functions and the initial treatment of the main impairments".

According to the document, eligible cases for admission to the abovementioned Units are: 
- patients coming from the Cardiac Intensive Care Unit due to a recent acute event (acute coronary syndrome with transfer within the first 5 days from the index event), and discharged with an early discharge program;

- patients coming either early from the Surgical Intensive Care Unit (within the first 5 days of the index event) or in the following phase who present complications (chronic phase with high care need);

- patients affected by severe ventricular failure and/or heart failure refractory to conventional therapy, requiring infusion support and continuous monitoring of hemodynamic parameters, making discharge and home management impossible.

These Units, which perhaps would be more appropriately defined as Units of Intensive and Sub-intensive Cardiac Rehabilitaton (UICR), may represent an adequate response to the management of these categories of patients whenever there is a plausible possibility of functional recovery and improvement of the quality of life. Hence, by UICR we mean a particular organizational structure and assistance path aimed at the functional recovery and clinical stabilization of critical and highly complex patients by means of rehabilitative, interdisciplinary cardiovascular medicine, including intensive interventions and diagnostic activities. These units thus require technology for continuous, also invasive, monitoring of vital functions; they must have a multidisciplinary staff (nurse, physiotherapist, dietician, psychologist) who, coordinated by a cardiologist, are able to develop a program of medical stabilization, early physiotherapy and intensive nursing aimed at close clinical monitoring, prevention of infections, early mobilization, nutritional optimizaton, cognitive re-education and the recovery of affective-relational aspects together with the caregiver, with the goal of weaning from dependence, preventing deconditioning and the complications related to immobility.

The UICR can take in patients both from acute intensive care settings, as described above, and from ordinary rehabilitation hospital units in the case of patients undergoing an episode of instability, who can find appropriate care within the UICR, unless treatments are required that can be administered only in conventional Intensive Care Units.

The need for the UICR is, therefore, not new - simply it has been largely disregarded within the national territory.

Even though in the ISYDE study of 2013 [17], of the 3527 beds registered, 408 (about 11\%) were described as "sub-intensive", it should be emphasized that this dynamic represents a spontaneous mode by which the CR structures have tried to fill an unmet medical need. There is in fact no tariff differentiation for the admission of the patients described above compared to those who access "conventional" CR pathways, nor any threshold indicated for the length of stay of patients in such sub-intensive beds. It would certainly be opportune to remedy this delay given that the epidemiological and demographic evolution and technological advances (allowing patients to successfully overcome increasingly critical clinical phases) mean that cardiology will more and more need to deal with cardiac patients presenting severe disabilities in the post-acute phase of ever-growing complexity.

\section{Outpatient, home and "digital" Cardiac Prevention and Rehabilitation}

Evidence on the benefits of structured secondary prevention programs, also confirmed by the recent guidelines, as well as the abovementioned epidemiological changes, make it increasingly necessary to differentiate the clinical settings for CPR according to the needs of the individual patient and for a more appropriate use of CR. Today, in fact, an undifferentiated CR is no longer suitable for patients with different pathologies, needs and objectives [40,41].

\section{Outpatient Cardiac Prevention and Rehabilitation}

The outpatient model, complementary to the inpatient model, can contribute to:

- make CR services more widespread, overcoming the limits of a national reality characterized by strong imbalances in the territorial distribution of dedicated presidia;

- favor continuity of care with rapid enrollment after discharge of patients with lower residual clinical risk;

- facilitate the recommendation of a CR program by acute-care cardiologists in those patients who, after cardiac surgery, have a low residual clinical risk.

The identification of the appropriate rehabilitation setting is today defined by consensus documents that favor a rational and appropriate use of the services offered. To this end, a description of both the confines and the contents of outpatient CR is in order.

Care settings in CR are constantly evolving, focusing on the outpatient regime for patients with a moderate risk profile, who are delivered "packages" of services, whilst maintaining the inpatient setting for patients at high risk and/or with residual instability, greater complexity and disability before or after the index event.

Outpatient CR has far more heterogeneous care models than inpatient $\mathrm{CR}$, both in relation to the type of patients it receives and to the therapeutic goals. It includes "simpler" facilities predominantly of secondary prevention, also directly linked to acute cardiology, and facilities also for complex patients which offer all the components of the CR. The outpatient intervention sometimes follows an initial phase of hospitalization and is more focused on physical training, modification of cardiovascular risk factors, optimization of therapy, health education and counseling, thanks also to the availability of a different temporal extension of the intervention. Just as we find, in inpatient CR, some facilities, in some cases equipped with sub-intensive therapies, that can manage situations of high complexity of clinical care and others more suited for the treatment of patients with less complexity, the same distinction also exists for outpatient CR, which can have:

- outpatient facilities of low complexity (secondary prevention clinics);

- outpatient structures able to provide complex CPR pathways (in some Regions still classified within a day-hospital regime, in others part of integrated outpatient pathways such as, for example, the Lombardy outpatient regime of Complex Outpatient Macroactivity). Outpatient CR can therefore be organized according to models of different intensity, but in order for them to be defined as "Cardiac Rehabilitation" interventions, specific structural, organizational and management requirements must be respected [40].

The facility must be equipped with a Rehabilitation Facility Plan that defines ex-ante its characteristics, the types of services offered, the work potential and professional figures involved, the admission procedures and links with other structures, and its compliance with the regional accreditation criteria (Table 3 ).

The personnel involved in routine clinical practice varies greatly, in relation often to local or organizational needs. Nevertheless, it is recommended that facilities be able to access, also functionally, those professionals (nurse, physiotherapist, occupational therapist, dietician, psychologist and also pharmacist, social worker, various specialists) (Table 4) that express the specificity and multidisciplinarity of CR. In fact, outpatient CR must be able to deliver the set of actions that constitute the "core" of CR, whose components, as indicated by the Scientific Community [2], have been reported previously. But we recall them here for the sake of reinforcement:

- Prognostic stratification and clinical stabilization,

- Counseling on physical activity, 
- Prescription of a personalized physical training program,

- Counseling on diet and nutrition,

- Body weight management,

- Management of dyslipidemia,

- Blood pressure control and management,

- Smoking cessation,

- Psychological and social intervention.

In general, outpatient CR programs are intended for more autonomous and stable patients who do not have any logistic-social needs warranting an inpatient program. The programs have a high intensity of rehabilitation but at a lower care intensity. The clinical complexity, which in the past was a non-coded selection criterion, does not represent as such the discriminating element between inpatient and outpatient CR, the modes of management of the disease, outcomes and complications having undergone profound changes (e.g. post-acute infarction care).

Therefore, patients who result at intermediate risk on risk stratification can be effectively followed by an outpatient treatment program, provided it is structured and complete, even if such patients may sometimes show clinical complexity, comorbidities or a concentration of risk factors demanding special attention. These patients must be followed in outpatient facilities able to provide complex care pathways.

For low-risk, stable patients, on the other hand, outpatient CR is essentially focused on preventive and educational aspects and can be de- livered in low-complexity outpatient settings (secondary prevention clinics).

Finally, there has been a progressive evolution of the organizational/administrative modalities of rehabilitative services: outpatient CR interventions are today articulated as a set of individual single services, or as service or day-service packages, with very variable architecture depending on the characteristics of the patients who access them and the different rehabilitation needs. In any case it is essential that patients can access the outpatient CR programs from the acute hospital or from an inpatient CR facility (in this case, as an extra-hospital continuation of the rehabilitation program) on the specialist cardiologist's prescription or, if referred from the local community, on the GP's prescription.

\section{Home Cardiac Rehabilitation}

CR, according to the latest guidelines, in its various clinical and organizational contexts, confirms its role as the standard of care through its multidisciplinary programs and as the most effective model for the implementation of secondary prevention. But one of its key problems is the lack of participation in rehabilitative programs due to the low number of structures in some regions, as previously mentioned, and to the difficulty especially of younger patients to adhere to inpatient pro-

Table 3. Contents of the Cardiac Rehabilitation plan for outpatients.

- Definition of the access groups with index event and assessment of complications of the acute phase and of comorbidities.

- Rehabilitation phase: post-acute, chronic stabilized

- Objectives to reduce clinical risk, manage comorbidities and disability.

- Assessment tools used.

- Structured educational programs for lifestyle change (smoking cessation, appropriate nutrition, physical activity, weight control, anxiety and depression).

- Prescription of physical activity to reduce the disability due to heart disease, improve functional capacity and promote social and work reintegration.

- Estimated duration of the rehabilitation program consistent with the goals set for each patient, and differentiated according to clinical and functional condition, and specific needs.

- Evaluation and path of organizational and clinical appropriateness and outcome indicators used.

- Periodic verification during the course of the program (interruption, transfer to acute ward, complications, etc.).

- Professional figures involved.

Table 4. Main contents of multiprofessional interventions implemented in the outpatient setting.

\begin{tabular}{|c|c|c|c|}
\hline Nurses & Physiotherapists & Dieticians & Psychologists \\
\hline $\begin{array}{l}\text { - Educational intervention } \\
\text { and verification of knowledge } \\
\text { about risk factors and lifestyle } \\
\text { changes to make } \\
\text { - Instruction and verification } \\
\text { of adequate capacity to } \\
\text { manage pharmacological } \\
\text { therapy (use of therapy cards, } \\
\text { dispenser, pillbox with timer) } \\
\text { - Instruction and verification } \\
\text { on self-monitoring and } \\
\text { self-care skills } \\
\text { - Learning and verification } \\
\text { of environmental strategies } \\
\text { with respect to residual } \\
\text { cognitive resources } \\
\text { - Educational intervention } \\
\text { to patient and caregiver } \\
\text { and its verification }\end{array}$ & $\begin{array}{l}\text { - Aerobic training with } \\
\text { incremental load } \\
\text { - Exercises to increase } \\
\text { strength and endurance } \\
\text { - Verification of patient's } \\
\text { capacity to self-manage } \\
\text { the physical exercises } \\
\text { performed at home } \\
\text { - Educational intervention } \\
\text { to patient and its verification } \\
\text { - Prescription of the physical } \\
\text { activity to be carried out } \\
\text { as a requirement for work } \\
\text { reintegration }\end{array}$ & $\begin{array}{l}\text { - Instruction and verification } \\
\text { on correct eating habits } \\
\text { - Management and evaluation } \\
\text { of goals agreed with food diary } \\
\text { and recall } \\
\text { - Educational intervention } \\
\text { and verification } \\
\text { - Training in self-monitoring } \\
\text { of food intake } \\
\text { - Support and practical guidance } \\
\text { to the caregiver }\end{array}$ & $\begin{array}{l}\text { - Verification of mood condition } \\
\text { with patient/caregiver } \\
\text { - Verification of acquisition } \\
\text { of adequate awareness } \\
\text { of the disease } \\
\text { - Verification of abstinence } \\
\text { from smoking and alcohol and } \\
\text { motivational reinforcement } \\
\text { for an adequate control of risk } \\
\text { factors } \\
\text { - Relaxation training } \\
\text { and indications for a correct } \\
\text { management of stress } \\
\text { - Activation of continuity of care } \\
\text { with referral to Community } \\
\text { Services for further diagnosis } \\
\text { and/or care, including welfare } \\
\text { assistance for socio-economic } \\
\text { problems }\end{array}$ \\
\hline
\end{tabular}


grams. Another fundamental point is the long-term continuation of the rehabilitation program at the time of discharge from the inpatient or outpatient setting: it is important that the patient enter into a virtuous circle, with the possibility of continuing the physical exercise and lifestyle changes implemented in a supervised context, so as not to lose the benefits achieved during the inpatient and outpatient rehabilitation phase, or even to obtain a further improvement.

Home rehabilitation can thus become not only an alternative for the less compromised and lower risk patients, but the natural long-term continuation of the rehabilitation process for effective secondary prevention. A systematic review of home and hospital rehabilitation trials did not reveal differences between the two settings in terms of outcome and adherence to therapy [42]. The advent of new information technologies and the widespread use of smartphones and of the numerous and diversified communication and videoconferencing systems, even through social network platforms, can allow an effective, efficient and quality remote clinical support inasmuch as it is managed by the same rehabilitation facilities. Home rehabilitation, thus configured (telerehabilitation) also allows the monitoring of many vital parameters, which, together with the transmission of ECG, can be of great support for the execution of an effective rehabilitation path and/or for structured secondary prevention programs [43]. Another important aspect, present in all the reports in the literature, is the improvement of the patient's quality of life and the patient's satisfaction about being followed at home. In fact, for an elderly person, sometimes not autonomous, moving from their home to reach the reference hospital may constitute a significant problem, and the use of telemedicine systems can be a promising option for rehabilitation activities carried out over a long period of time.

\section{"Digital" Cardiac Prevention and Rehabilitation: Digital tools}

The aging of the population, together with the growing impact of chronic conditions and multimorbidity underlie the steady increase in the demand for healthcare services. To succeed in this enterprise, health systems must pursue innovative solutions through new technologies and changes at the organizational level. We can identify some basic points:

- the configuration of new care models;

- the use of Health Technology Assessment to obtain a better quality and sustainability of health services;

- the involvement of multidisciplinary healthcare teams with new or redefined roles for the members;

- active cooperation between health professionals and patients;

- the use of digital solutions to guarantee efficient and cost-effective assistance;

- the use of health-related personal data and of digital clinical records.

Further supporting the digital services infrastructure for e-Health will also serve to promote the use of data for public health and for research, and allow us to move closer to the concept of personalized medicine. In particular, by using digital solutions, such as wearable devices and mobile health applications (m-Health), citizens can actively participate in health promotion and self-manage some aspects of their chronic disease. The development of digital tools capable of strengthening citizen empowerment and enhancing the centrality of people in the care process therefore appears to be mandatory. The use of common standards will improve the understanding of health and disease, allowing, moreover, to prevent diseases more effectively, speed up diagnosis, develop better prevention and treatment measures and monitor their effectiveness and possible undesirable effects.

CPR fits very well into the path of development and use of e-Health and m-Health. Just think how the growing multiplicity of "wearable" sensors of biological signals in normal life and their remote monitoring can be of great help in the management of the so-called Phase 3 of CPR. This phase corresponds to the long-term outpatient management of cardiac patients, its goal being to provide secondary prevention services to the individual and the community. Moreover, it is precisely in the long term that the problem of adherence to the correct prescriptions (both pharmacological and those regarding lifestyle) manifests itself fully, and where there is a need for new paths and methodologies to strengthen the patient's empowerment.

\section{Minimum operational criteria to define a structured intervention of secondary cardiovascular prevention (secondary prevention service)}

The clinical, organizational and structural requirements for inpatient CR structures have been extensively described in previous consensus documents [40]. This section proposes an update of the minimum requirements, i.e. the technological and human resources necessary for the correct implementation of secondary cardiovascular prevention programs $[2,40]$.

Secondary cardiovascular prevention, a subspecialty of clinical cardiology that deals with cardiac patients in the post-acute phase, is essential and strongly recommended in those highly prevalent diseases, in particular ischemic heart disease, heart failure and atrial fibrillation, that also have the highest incidence of events and relapses and the greatest risk of low adherence to treatment due to the complexity of the pharmacological strategies they require [27].

In addition to these, degenerative heart valve disease and atrial fibrillation are predictors of destabilization of the clinical picture, of new hospitalizations and of increased cardio-embolic risk, especially atrial fibrillation with regard to this last aspect. Special attention must also be paid to subjects with recurrent events and/or with generalized atherosclerosis.

First of all, it is essential that patients at high risk of recurrence after an acute event are correctly identified. Outpatient facilities must operate harmoniously at least with one acute-care cardiology ward in order to guarantee diagnostic and care continuity and functional linkage with other specialties and services. It is necessary to guarantee a dynamic but structured link with the local territory (with slots open to general medicine and/or other specialties) for the assessment/treatment of patients in secondary prevention/rehabilitation pathways. The ideal system is of a circular, open and dynamic type, which responds to the stages of acute phase, chronic phase, stabilization/destabilization and to the complexity of the clinical and pharmacological management. In this movement, the patients are welcomed after an acute event and returned with precise indications to the GP in the phase of stabilization, to then return for programmed periodic controls or in the case of clinical destabilization. The indicated care pathways must adhere to the guideline indications and must be explained in the clinical report sent to the GP.

Table 5 summarizes the minimum technological and staff requirements in relation to the components of each intervention and activities to be performed.

The first evaluation is the patient history and clinical assessment (Table 5). Patients with recent acute coronary syndrome should be stratified using the risk scores best adapted to the clinical situation starting from the index event (Killip class; TIMI risk score, GRACE at 12 months). It is necessary to know the course of this in detail in order to determine if the patient is at high clinical risk. The secondary prevention intervention should be aimed at: reducing the risk of recurrence of ischemic events, preventing post-infarct myocardial remodeling and the evolution towards heart failure or its relapses; preventing sudden 
Table 5. Main work components required to implement a structured intervention of Secondary Cardiovascular Prevention in Cardiac Rehabilitation.

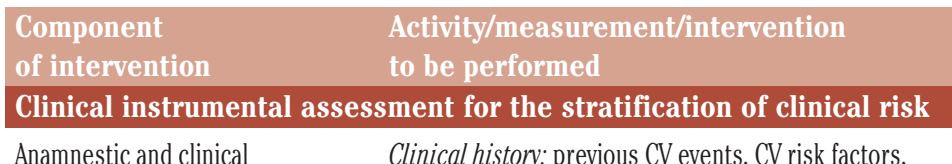

Anamnestic and clinical evaluation of the patient (stratification of residual risk)

comorbidities, familial predisposition for early CVD,

level of physical activity and degree of sedentariness,
Instrumental evaluation (CV risk stratification) education and social situation

Physical examination: weight, height, BMI, BSA, waist circumference, complete cardiological examination, measurement of $\mathrm{HR}$ and $\mathrm{BP}$

Current symptoms: NYHA class and/or CCS

Evaluation of the index event (AHF/AMI), HF,

arrhythmic events/AF

- High Killip class at admission

- $40 \% \mathrm{EF}$

- Patients with EF in the intermediate range associated with a remodeling predictor (one of: mitral insufficiency $>1$, restrictive diastolic filling, high asynergy score and non-dilated ventricle)

- Important variation of BNP/use of loop diuretics

- DM

- Peripheral arterial disease

- History of angina or previous AMI

- Multivessel coronary disease/incomplete or non-revascularized revascularization

- Type, site and number of implanted coronary stents Evaluation of risk indices and/or risk scores when applicable (clinical and atherothrombotic residual risk, GRACE score at 12 months, DAPT score or DAPT precise score (AHF), REACH, CHA2DS2-VASc score (AF), HAS-BLED Dutch lipid score (detection of familial forms of dyslipidemia), arrhythmic risk, indication to device implants (CRT/ICD)

Aims:

- Stratification of residual risk

- Formulation of personalized secondary prevention programs for the patient.

ECG: HR, rhythm, QRS, conduction disorders, ventricular repolarization

Echo-color Doppler M-mode 2D: evaluation of LV geometry and function, LV mass; diastolic function: transmitral flow, right superior pulmonary vein, septal and lateral mitral TDI, valvulopathies (quantification of morphology and function, calcifications, extent of valvular defect), aortic vessel, left atrium (linear dimensions and volume adjusted for BSA) right ventricle and pulmonary pressure. Presence of pleuro-pericardial effusion. Echo-Doppler TSA, aorta and peripheral vessels (when indicated) ECG Holter/stress ECG (when indicated)

Exercise stress echo (when indicated) Angio-CT/MRI and/or nuclear medicine (when indicated)

\section{Minimum work technology Personnel}

required required

Suitable environments for the clinical examination, physical examination and interviews $\mathrm{PC}$ and internet connection Database with data storage system (preferably online network connection with

CR centers)

Possibility to review instrumental imaging examinations

Complete reports of the index event [coronarography and coronary interventional procedures viewable (CD)] with possibility to save and store exam
Clinical cardiologist with expertise in prevention and rehabilitation Physiotherapist Physical therapist/psychologist Nurse with training in counseling and CV prevention 12-lead electrocardiograph ECG Holter Color Doppler 2D echocardiograph, TDI with the possibility of recording and digital storage for review and intra-patient comparison. Functional linkage with angiology/vascular surgery/radiology and/or nuclear medicine for level II examinations
Clinical cardiologist with expertise in prevention and rehabilitation Expertise in echocardiography and $\mathrm{CV}$ imaging

Nurse

Functional link with radiologist/nuclear physician/angiologist

Stratification of the associated CV risk (lipid targets, BP) and adherence to evidence-based therapies

Laboratory assessment
Laboratory tests: blood count, renal function, electrolyte panel; blood glucose (HbAlc, if abnormal or diabetic), total cholesterol, HDL, triglycerides, LDL cholesterol, non-HDL cholesterol (if triglycerides $>300 \mathrm{mg} / \mathrm{dl}$ ), iron test (if $\mathrm{Hb}<9 \mathrm{~g} / \mathrm{dl}$ )

Hepatic battery, myoglobin, CPK, thyroid profile
Link available with laboratory

Nurse analysis 
Table 5. Continued from previous page.

\begin{tabular}{|c|c|}
\hline $\begin{array}{l}\text { Component } \\
\text { of intervention }\end{array}$ & $\begin{array}{l}\text { Activity/measurement/intervention } \\
\text { to be performed }\end{array}$ \\
\hline $\begin{array}{l}\text { Lipid profile } \\
\text { (stratification of CV risk) }\end{array}$ & $\begin{array}{l}\text { Evaluation: lipidological objective examination (corneal } \\
\text { arch, xanthomas, xanthomas), lipid profile, possible } \\
\text { integration with apo-B, apo-A, Lp (a), homocysteinemia, } \\
\text { MTHFR, Dutch Lipid Score (assessment of familial forms } \\
\text { in secondary prevention and possible family screening) } \\
\text { Management of statin intolerance } \\
\text { Aims: } \\
\text { - Diet modification, physical activity and prescription } \\
\text { of drug therapy } \\
\text { - High intensity/high dose statin prescription; } \\
\text { association of high-intensity statin with ezetimib; } \\
\text { recognition of patients eligible for prescription } \\
\text { of anti-PCSK9 according to indications. } \\
\text { - LDL cholesterol <55 mg/dl in subjects at very high } \\
\text { risk, <70 mg/dl in subjects at very high risk or } \\
\text { a reduction } \geq 50 \% \text { if the target cannot be reached. } \\
\text { In this case, evaluate the possible addition } \\
\text { of anti-PCSK9 } \\
\text { - LDL cholesterol }<100 \mathrm{mg} / \mathrm{dl} \text { in high-risk subjects } \\
\text { - LDL cholesterol }<115 \mathrm{mg} / \mathrm{dl} \text { for subjects } \\
\text { at moderate or low risk } \\
\text { - Total cholesterol } \leq 190 \mathrm{mg} / \mathrm{dl} \\
\text { - Triglycerides }<150 \mathrm{mg} / \mathrm{dl} \\
\text { - Non-HDL cholesterol }<100 \mathrm{mg} / \mathrm{dl}\end{array}$ \\
\hline
\end{tabular}

BP Assessment: resting BP, self-monitoring and/or ABPM (stratification of CV risk) to evaluate masked hypertension or white coat effect. $\mathrm{BP}$ during exercise when stress hypertension is suspected. Check for subclinical organ damage at cardiac and vascular level Aims:

- BP 120-129/70-79 mmHg in patients with $\mathrm{DM} / \mathrm{HMOD} / \mathrm{IHD} / \mathrm{stroke} / \mathrm{very}$ high risk

- BP 130/139/70-79 mmHg for age > $>5$ years/chronic renal failure

DM (stratification of CV risk)

Lifestyle modification

Smoking cessation
Assessment: fasting glucose control; HbAlc; load curve (if indicated), BP at rest, self-measured and/or ABPM to evaluate masked hypertension or white coat effect. Investigate for subclinical organ damage at cardiac and vascular level

Aims:

- Glycemic control ( HbAlc $<7.0 \%$ ( $<53 \mathrm{mmol} / \mathrm{mol})$

- Lipid profile: LDL cholesterol $<55 \mathrm{mg} / \mathrm{dl}$ in subjects with DM at very high risk, $<70 \mathrm{mg} / \mathrm{dl}$ in subjects at very high risk or a $\geq 50 \%$ reduction if the target the possible addition of anti-PCSK9

- LDL cholesterol $<100 \mathrm{mg} / \mathrm{dl}$ in all other subjects with DM

- BP 120-129/70-79 mmHg cannot be reached. In this case, evaluate

\section{Minimum work technology Personnel} required required

Possibility of

prescribing/performing blood chemistry tests

Connection also in network with lipidological reference center to refer selected cases and/or for genetic diagnosis
Clinical cardiologist with expertise in prevention and rehabilitation Expertise in the etiological evaluation of dyslipidemia and pharmacological management (SAMS, statin intolerance, drug interactions) Nurses with training in counseling and CV prevention (co-management of adherence)
Sigmomanometer Different bracelets according to body size Device for ABPM or functional link with Cardiology service equipped with this device
Clinical cardiologist specialized in prevention and rehabilitation Nurse with training in counseling and CV prevention
ABPM availability for monitoring blood pressure profile
All smokers should be encouraged in a professional manner to stop smoking permanently by avoiding all forms of tobacco and smoking methods (electronic cigarettes)

Follow-up, referral to special programs and/or pharmacotherapy (including nicotine replacement therapy) is recommended

Ask the patient about their smoking status and the use of other tobacco products, specify quantities (cigarettes/day) and duration of smoking (number of years), use structured approaches, e.g. 5As: Ask, Advise, Assess, Assist, Arrange

Intervention: offer nicotine replacement therapy (bupropion, varenicline) if not contraindicated Aim: long-term smoking cessation
$\mathrm{PC}$ and internet connection Database with data storage system for follow-up management (preferably connection online with other centers in CR network) Contact with an anti-smoking center and/or possibility of links with specialists in the sector (behavioral and group counseling or individual counseling)
Clinical cardiologist specialized in prevention and rehabilitation Nurse with training in counseling and CV prevention (co-management of adherence) 
Table 5. Continued from previous page.

\section{Component of intervention}

Diet/weight management

\section{Activity/measurement/intervention} to be performed

Nutritional assessment: dietary and clinical history, anthropometry, biochemical and instrumental data, physical examination for nutritional status, evaluation of nutritional awareness and eating habits Characteristics of healthy eating

- Saturated fatty acids: less than $10 \%$ of the total energy supply, substituting them with polyunsaturated fatty acids

- Trans-unsaturated fatty acids: as little as possible, preferably not from processed foods and accounting for less than $1 \%$ of total energy input from natural sources.

- Less than $5 \mathrm{~g}$ of salt per day

- 40-45 g of fiber per day, preferably from integral products

- $>200 \mathrm{~g}$ of fruit per day (2-3 servings)

- >200 g of vegetables per day (2-3 servings)

- Fish 1-2 times a week, one of which consisting of fatty fish

- 30 g unsalted nuts a day

- Consumption of alcoholic beverages: to be limited to 2 glasses per day ( $20 \mathrm{~g} /$ day of alcohol) for men and 1 glass a day ( $10 \mathrm{~g} /$ day of alcohol) for women

- Consumption of sugary and alcoholic drinks should be absolutely discouraged

Both overweight and obesity are associated with an increased risk of CV-related and all-cause mortality. All-cause mortality is lower in the presence of a BMl $20-25 \mathrm{~kg} / \mathrm{m} 2$ (in subjects aged $<60$ years); greater reductions in body weight do not have a protective effect against CVDs.

- The ideal body weight in the elderly is higher than that of a young or middle-aged person. If the waist circumference is $\geq 80 \mathrm{~cm}$ in women and $\geq 94 \mathrm{~cm}$ in men, suggest changes in lifestyle and consider strategies for treatment of the metabolic syndrome

Aims:

- For overweight or obese individuals, it is recommended to achieve an ideal body weight (or weight reduction) in order to reduce blood pressure, dyslipidemia and the risk of developing type $2 \mathrm{DM}$.

Adherence to therapy
Adherence: to drugs and self-monitoring (weight, BP, symptoms), family support to support treatment adherence
Education: clear and comprehensible information on the aims of the CR and secondary prevention program (correction of risk factors, significance and importance of adherence to evidence-based therapies in terms of prognostic effects on the ischemic and atherosclerotic residual risk) Teach-back: education on self-monitoring (of weight, BP, warning signs and symptoms of clinical instability) and on self-management of heart failure therapy

\section{Minimum work technology Personnel} required required

Balance, centimeter for waist circumference measurement Availability of nutritional and dietary advice

Nurse with training in counseling and prevention of CV (co-management of adherence) Availability of consultation with a nutritionist for referral of patients to the dietician
PC and internet connection Database with data storage system for follow-up (preferably with online connection with the $\mathrm{CR}$ network)
Clinical cardiologist specialized in prevention and rehabilitation Nurses with training in counseling and CV prevention (co-management of adherence)

2D, 2-dimensional; ABPM, ambulatory blood pressure monitoring; apo; apolipoprotein; BMI, body mass index; BNP, brain natriuretic peptide; BSA, body surface area; CCS, Canadian Cardiovascular Society; IHD, ischemic heart disease; CPK, creatinphosphokinase; CR, cardiac rehabilitation; CRT, cardiac resynchronization therapy; CV, cardiovascular; DM, diabetes mellitus; ECG, electrocardiogram; AF, atrial fibrillation; HR, heart rate; EF, ejection fraction; Hb, hemoglobin; HbAlc, glycated hemoglobin; HMOD, hypertension-mediated organ damage; ICD, impiantable cardioverter-defibrillator; AMI, acute myocardial infarct; Lp(a), lipoprotein (a); CVD, cardiovascular disease; MTHFR, methylenetetrahydrofolate reductase; NYHA, New York Heart Association; BP, blood pressure; PCSK9, proprotein convertase subtilisin/kexin type 9; MRI, magnetic resonance imaging; SAMS, statin-associated muscle symptoms; HF, heart failure; ACS, acute coronary syndrome; CT, computed tomography; TDI, tissue Doppler imaging; SAT, supra aortic trunk. 
death from complex arrhythmias by evaluating the progression of left ventricle remodeling/dysfunction; verifying if there is an indication for a device implant (formulation over time of the indication for resynchronization therapy or defibrillator implant); preventing risk of stroke through early recognition of potentially thromboembolic atrial arrhythmias. It is necessary that the clinic be coordinated by a clinical cardiologist with expertise in prevention and rehabilitation, constantly updated on the management strategies for cardiovascular risk factors and residual clinical risk, and on the management of medications and drug interactions. The minimum operational criteria for a complete assessment of secondary cardiovascular prevention, generally performed in an outpatient setting, require as part of the technological equipment at least one PC with a data storage system (database dedicated to secondary prevention). There must be available at least a basic system for reviewing instrumental examinations and managing diagnostic imaging (coronary angiography and intervention procedures of the index or subsequent event, computerized tomography or magnetic resonance), better if able to manage also the echocardiograms for intra-patient comparison. Dedicated personnel is required, or at least trained personnel, and basic counseling and systematic collection of data should be performed in the most standardized way possible.

The technological requirements are reported in Table 5 . Instrumental diagnostics is necessary, together with the clinical documentation of the index event, for the re-assessment of the risk. For example, when identifying the patient at high atherothrombotic risk, it is necessary to verify the extent of coronary atherosclerosis (review of the coronary angiography), the type of revascularization intervention (number, complexity, type of stent). Patients at risk for the presence of vasculopathy in other vascular districts in addition to the coronary one must be identified, planning over time the diagnostic workup, e.g. on the carotid, peripheral and aortic vessels.

Parallel to the clinical assessment with diagnostic imaging, cardiovascular risk stratification involves an assessment of the associated cardiovascular risk, i.e. those parameters that represent risk exposure (lipid structure, blood pressure, diabetes) (Table 5). Presence of a family history of dyslipidemias should be assessed as well as the achievement of the intervention targets. In this case, the basic technological equipment must also include the availability of devices (at least one) for 24-h monitoring of blood pressure (also in functional link with the cardiology department) for the evaluation of blood pressure control, the diagnosis of white coat or masked hypertension, especially useful in patients with diabetes mellitus who often show prognostically adverse phenomena of the pressure phenotypes (e.g. morning rising, reverse dipper or extreme dipper). The clinical cardiologist with expertise in prevention and rehabilitation must be familiar with the use of the main hypoglycemic drugs, at least in terms of their possible interactions or side effects with cardioactive drugs, in order to be able to co-manage the patient with the diabetologist. The secondary prevention clinic must have a close and collaborative contact with the diabetologist, or be able to offer this service to patients who have not been previously referred for such controls. In any case, patients with suspected pre-diabetes or metabolic syndrome should be encouraged and/or helped to undergo a first diabetic check-up to be able to establish the diet and/or therapy as soon as possible (Table 5). The secondary prevention clinic must be in close contact and collaboration, besides with diabetology, also with other specialties (pneumology, nephrology) that represent prevalent comorbidities in cardiac patients, or be able to offer it, facilitating access to patients who have not previously undergone these controls (Table 5). At the same time as prescribing pharmacological therapies aimed at reaching the targets, the secondary cardiovascular prevention clinic is the place where lifestyle changes are promoted through the combined action of the cardiologist and nurse, who provide at least (minimum criterion) a basic counseling with particular regard to physical activity, avoiding to generate resistance and referring patients from time to time to multispecialistic interventions if deemed more suitable or if not practicable in situ (Table 5). Besides controlling the achievement of the targets recommended by the international guidelines, with particular attention to heart rate, blood pressure, LDL cholesterol, glycated hemoglobin (in diabetic patients), smoking cessation, body weight and physical activity, it is of great importance to systematically evaluate the adherence to prescribed therapies and the maintenance of the therapeutic alliance between the physician and the patient (Table 5).

In particular, at each check-up the following must be evaluated:

- therapeutic appropriateness and prescription of prolonged dual antiplatelet therapy over 12 months in patients at high thrombotic-ischemic risk;

- therapeutic appropriateness and prescription of short-term dual antiplatelet therapy (from 1 to 3/6 months) in patients with a combination of double anti-aggregation and anticoagulation in relation to the balance between thrombotic and hemorrhagic risk (comorbidity) and/or the need to undergo non-cardiac surgery;

- therapeutic appropriateness and prescription of new anticoagulants and management of anticoagulant therapy in complex patients, patients with comorbidities, or frail patients;

- management of statin/ezetimib therapeutic adherence according to guidelines;

- prescription therapy of PCSK9 inhibitors;

- therapeutic suitability of prescription of sacubitril/valsartan;

- persistence of therapy in all the categories described above.

\section{Cardiac Prevention and Rehabilitation and treatment adherence}

Adherence to medical treatment is the key to success in almost all chronic conditions, especially in cardiovascular diseases where it plays a decisive role; the first references to the adherence problem date back to the ' 60 s [44], but the problem is more current than ever and at the center of a lively scientific debate.

Adherence is defined by the WHO as the behavior of a subject/patient regarding drug intake, observance of a diet, or of a particular lifestyle that corresponds to the recommendations agreed with a health professional [45]. The need to clearly define adherence arises from the fact that all too often it is used as a synonym of compliance which, instead, indicates a passive process of the patient compared to the prescriber, placing the two figures on asymmetrical and distant planes, a situation absolutely to avoid [46]. Vrijens et al. [47] have expanded the concept of adherence to pharmacological therapy by characterizing it as a continuous process consisting of three phases: initiation, implementation and interruption. The initiation of treatment is the administration of the first dose of drug, followed by the implementation, that is the continuous intake of the prescribed dosage, and it ends with the interruption, which decrees the end of therapy. In these terms, lack of adherence can occur at all levels, as a delay or lack of initiation of therapy, as a suboptimal implementation of the dosing regimen, and finally as a premature termination of treatment.

The factors underlying the lack of adherence are many and they can be divided into those related to the patient, the prescriber, the health system and finally the environment [48]. First, those referable to the patient are: age, to which a greater incidence of comorbidity is often correlated [49], social status, negative attitudes about drugs and their adverse effects, and cultural and psychological/cognitive limitations. Lack of knowledge and awareness about the disease can lead to a passive attitude that gets worse if the patient becomes frustrated in not 
perceiving outcomes and benefits from treatment, which is why lipidlowering therapy generally has less adherence than antihypertensive therapy (46.9\% vs 57.7\%) [50]. As for the prescriber, this concerns not only the cardiologist but also the GP, as they both play a fundamental role in patient education, in their availability to discuss with the patient and in the choice of the most suitable therapeutic options for the individual patient - in a word communication [51]. An emblematic example of the importance of drug delivery methods is the meta-analysis conducted by Caldeira et al. [52], which showed that a single daily dose is associated with a $56 \%$ (RR 0.44; 95\% CI 0.35-0.54) reduction in the risk of non-adherence (i.e. greater adherence) compared to multiple administration during the day [52]. Another phenomenon attributable to the prescriber is the so-called prescriptive cascade, i.e. situations in which the side effects of a therapy are erroneously interpreted as symptoms of a further pathological process, followed by further prescription. Therefore, the prescriber must always have a careful and critical attitude in regard to polytherapies in order to simplify and optimize them with the new opportunities offered by the current pharmacological/pharmaceutical scenario [53].

The Italian healthcare system impacts to a lesser extent on non- or reduced treatment adherence compared to the US, as it has a system of reimbursement and/or sharing of the pharmaceutical costs, particularly significant for patients with chronic diseases. The same approach exists with regard to access to health facilities for planned follow-up or in the event of an emergency. The result is a lower economic burden in Italy than in the U.S. of long-term pharmacological treatments, and this could favor adherence. Finally, among the environmental and social determinants of adherence there can be many variables such as accessibility to the drug or its prescription, the individual's social condition, the influence of the mass media and, recently, the introduction of generic drugs that often change the packages, so confusing the patient.

The size of the problem is well described by a meta-analysis performed on 20 studies involving over 370,000 patients, which observed that adherence to drug therapy for cardiovascular drugs is only $57 \%$ (95\% CI 50 64) after a median 24 months from the prescription, although there is greater adherence in secondary prevention (66\%; 95\% CI 56-75) than in primary prevention (50\%; 95\% CI 45-56) [54]. It has been calculated that the poor adherence to therapy translates into about 200,000 deaths a year in Europe with an estimated cost of about 125 billion euros/year in Europe and 300 billion dollars/year in the U.S. [55,56]. Numerous published studies have documented the effects of adherence on the outcomes obtained in patients suffering from cardiovascular diseases. In a recent cohort study of 4015 patients with previous myocardial infarction stratified, according to adherence to angiotensin-converting enzyme inhibitors and statins, as fully adherent, partially adherent and non-adherent to therapy (respectively, if regular assumptions were $\geq 80 \%, 40-79 \%$, or $<40 \%$ ), a lower incidence of major cardiovascular events was observed among those with complete adherence to therapy compared to partially adherent [18.9\% vs 24.7\%; hazard ratio (HR) 0.81 ; IC 95\% 0.69-0.94]; the difference was even more significant for non-adherent patients (18.9\% vs. $26.3 \%$, HR $0.72,95 \%$ CI 0.62-0.85) [57]. What has been said so far is valid in any area of cardiovascular disease, especially in chronic heart failure, in which adherence to therapeutic indications, whether pharmacological or related to the daily lifestyle, significantly reduces the readmissions for heart failure [58,59].

Although, as we have seen, the poor adherence to drug therapy has a significant impact on the prognosis of patients, we must not forget that this represents only one aspect. A change in lifestyle must be associated, with particular attention to a correct diet, regular physical activity and smoking cessation.

A substantial contribution to achieving and maintaining adherence, understood in the broadest and fullest sense of the term, can be offered by the CR programs, not by chance defined as the union of all the interventions required to guarantee physical and psychological improvement and social treatment of patients with chronic disease or following an acute cardiovascular event. CR, through specific core component interventions [2], is able to significantly reduce the smoking habit, improve dietary habits, increase the habit of regular physical activity, promote the titration of the recommended drugs up to the effective doses in randomized trials, or in any case up to the maximum tolerated dose and, finally, increase the adherence, as assessed by the Morisky scale (Morisky Medication Adherence Scale, MMAS); all these benefits are maintained over time, as demonstrated by the ICAROS study conducted in various Italian centers of CR [9]. The results reported in the ICAROS study translate into a concrete improvement of patients' lives, especially in terms of outcomes. In an important meta-analysis, Anderson et al. [13] evaluated 14,486 patients in 63 studies, with a median follow-up of 12 months, demonstrating that CR programs can reduce cardiovascular mortality and hospitalizations, improving the quality of life of patients. A recent study was conducted in a cohort of patients who performed an outpatient CR program following cardiac ischemic events (myocardial infarction with and without ST-segment elevation), percutaneous or surgical revascularization (also associated with valvular surgery) with a 5-year follow-up [11]. The results that emerged are in line with the conclusions of the previously mentioned meta-analysis, demonstrating that the positive effect of CR, in terms of a reduction in hospitalizations and combined hospitalization and cardiovascular death, is significant and is maintained over time.

Based on the numerous and solid evidence, the ESC guidelines on cardiovascular prevention, give participation in CR programs a class I recommendation with a high level of evidence, for patients hospitalized for acute coronary events and/or undergoing coronary revascularization and for those suffering from heart failure [1]. CR must be understood as an opportunity to support and educate patients, which must be implemented in its different forms - inpatient, outpatient or through telemedicine.

For a true benefit to be obtained, it is of paramount importance that the patient be motivated to take charge of their condition so as to independently pursue a virtuous lifestyle path also after discharge. Excessive reliance on rehabilitation staff, by the patient, also after the CR program, can be detrimental if the patient has not reached an active and convinced attitude [60] at the end of the rehabilitation process. On the other hand, in order to pursue the good of the patient, offering them a considerable opportunity in their clinical path, it is essential to overcome the skepticism about the benefits of CPR, both on the part of the clinicians involved in the acute phase of the cardiovascular event, and by the patients themselves, who are often not motivated to undertake the proposed rehabilitation programs in a serious and constant manner.

\section{Specific aspects of Cardiac Prevention and Rehabilitation for the Elderly and Very Elderly}

The rehabilitation of patients with coronary heart disease, following a structural intervention, post-cardiac surgery, or with heart failure, will increasingly involve elderly patients (age $>75$ years) and be characterized by more complicated organ disease, greater comorbidity, the possible co-presence of functional and cognitive deficits, and emotional disturbances or social isolation. While some of these conditions represent a specific indication for rehabilitation, on the other hand, doubts about the usefulness and feasibility of offering rehabilitation programs to elderly patients that were originally designed for young and adult subjects in poorly compromised functional conditions has led to a limited prescription of $\mathrm{CR}$ for the older age classes up until recently. 
The term "elderly" refers to a very heterogeneous population: from subjects that are functionally intact to patients with cardiovascular risk factors/chronic organ diseases or severe and multiple comorbidities but without dysautonomia, up to the "complex" elderly. The "complex" elderly patient is one who presents one or more of the following conditions: disabilities pre-existing or secondary to the index event, changes in cognitive abilities, affective disorders, nutritional deficits and disadvantaged social/family/economic conditions. It follows that the design and implementation of the CR intervention must be extremely differentiated, taking into account the specific needs of the particular individual and focusing on the most significant interventions for each subject [61] (Table 6).

CR consists of an integrated multidisciplinary program, combining the skills of a multi-interdisciplinary team, with several key areas of intervention (Table 7), aimed at recovering and maintaining functional capacity and emotional wellbeing, limiting the progression of cardiovascular disease and preventing disability, non self-sufficiency and cognitive decline [62].

\section{Modes of multidimensional evaluation}

The multidimensional evaluation is a fundamental process that includes clinical, functional, emotional, cognitive and social domains, in order to select the most appropriate rehabilitation program for the elderly patient, with reasonable and clinically valid goals to pursue. The aim is to minimize the likelihood of complications and to promote adherence to the program, in order to obtain the greatest possible re- covery of autonomy in the activities of daily life. Many tools for basic assessment have been considered as tools of the specialist (geriatrist or psychologist), whereas instead they must become part of the skills of properly trained professionals. The field of CR, through its Scientific Society, has promoted the above process through specific courses aimed at its members, in order to disseminate skills and work capabilities in the national healthcare structures. The main characteristics of multidimensional evaluation in the elderly cardiac patient are listed in Table 8, while the modalities for performing the assessments are presented in Table 9 .

\section{Identification of the rehabilitation goals}

Functional status and quality of life. The maintenance of a good state of health is related to the integrity of the functional status: this relationship summarizes the set of physical and cognitive abilities that allow one to perform the normal activities of daily life and to maintain a sufficient social network. It thus expresses a central dimension both as an indicator of health and as a goal of care [63].

The decline of muscle mass and strength in elderly subjects, better defined as sarcopenia, is a risk factor associated with frailty: comorbidity, inflammation, depressive symptoms, disturbance of balance are among the many factors that intertwine in a complex way with cardiovascular health and old age in determining functional decline [64]. The loss of strength that accompanies the reduction of muscle mass causes need to make a greater effort in performing the usual activities, while multimorbidity and hospitalizations produce further functional

Table 6. Main domains of the treatment program for the elderly.

- Respect the subject's preferences

- Weigh up the evidence on the most important goals

- Estimate the clinical and functional prognosis

- Evaluate the clinical efficacy of the planned interventions

- Examine possible interactions of the therapies

- Reduce the risks of side effects

- Communicate

- Discuss the therapeutic options with the patient

Table 7. Principles of planning rehabilitation for the elderly.

- Identify the general and specific goals

- Carry out the evaluation with appropriate tools

- Select and implement appropriate programs

- Customize for each condition

- Design significant and sustainable interventions

- Involve the patient and respect their preferences and expectations

Table 8. Multidimensional assessment and goals in the field of rehabilitation.

- General clinical conditions, comorbidities (to identify and also correct with drug therapy)

- Disability, with validated tests

- Frailty, with validated tests

- Emotional and cognitive state, with specific tests (to detect and eventually treat anxiety, depression and cognitive decline)

- Aerobic capacity, motor function, balance (to define the goals of intervention at the functional level)

- Nutritional status and eating habits (protein-calorie malnutrition, including secondary to solitude, isolation, economic conditions, social deprivation, or to edentulism and hyporexia)

- Cardiovascular risk with impact on the cardiovascular prognosis significant for the elderly subject (implementation of a counseling program for: correction of risk factors, dietary-behavioral management, maintenance of physical activity at home)

- Social and family support (for the best adherence to rehabilitation programs and education for self-management of drug therapy) 
decline that increases the risks of disability and dependence, amplified by the possible co-presence of depressive symptoms.

Numerous studies on CR utilize, among the indicators of improved quality of life, some variables that measure the perceived disease severity and its impact on quality of life.

It has been shown that in the elderly who participate in rehabilitation programs, additional or different benefits are obtained with respect to those traditionally examined, such as an improvement in mental health, in pain perception or in the limitation determined by the disease status, with a significant impact on daily life.

Interventions to improve functional capacity. Many elderly people consider preserving the basic activities of daily life and maintaining independence as their main objectives, more important than the traditional assessment of the treatment outcomes of the single disease [65]. Exercise is able to produce effects of considerable importance, since the elderly employ a large share of their functional capacity to perform even simple daily activities. Since cardiovascular disease produces ab auto-limitation of activity, which causes the reduction of strength and muscle mass, CR programs must include aerobic exercise to increase exercise resistance and muscle strengthening exercises of the extensor muscles of the lower limbs, which support the ability to walk, get up from the chair and maintain balance, and/or of the upper limbs, useful for carrying out household chores. The greater the level of frailty or sarcopenia, the greater the potential benefit of improving physical performance on both the quality of life and prognosis.

Interventions for adherence to therapy. This subject has already been discussed in the previous section. On discharge from the acute departments, it is often left up to the patient to interpret what is meant in the pharmacological prescriptions reported in the discharge letter. The therapeutic scheme may not be understood and it sometimes may be arbitrarily combined with therapies previously taken in the past. This aspect, frequently ignored, is instead fundamental, especially for the elderly who were already taking prescription medicine before admission: at home there are frequent overlaps between usual care and the new prescription, with the risk of ineffectiveness, side effects or even serious complications.

CR, thanks to the interaction with the patient, staff skills and the opportunity for receiving adequate feedback, plays a fundamental role in guaranteeing the best therapy and promoting adherence: actions include a review of the pharmacological therapies, the selection of medicines that "must not be avoided", and identification of potential interactions or adverse reactions. The practical strategies are based on reducing the number of assumptions in favor of medicinal products in combination, using dispensers with daily preparation of therapy, a detailed illustration of the new therapy compared to the previous one, the

Table 9. Examples of some assessment modalities and tools for elderly patients with heart disease.

Functional capacity (in relation to the test to perform)

- Distance walked on the 6-min walk test

- Score on the Short Physical Performance Battery with analysis of gait speed, balance and strength of the lower limbs (chair standing)

- Performance on the cycle ergometer or treadmill

- Peak VO2 at cardiopulmonary test

- Strength of lower limb segments, with dynamometer

Perceived exercise tolerance

- Fatigue perception with Borg scale (RPE score 6-20)

- Intensity of dyspnea with Borg scale (CR10)

Quality of life

- Score relative to the instrument used, "generic" or "specific"

Cognitive status

- Screening, using the Mini Cog Test (repetition of three words and drawing of a clock with the hands pointing to the time as specified)

- Mini-Mental State Examination (MMSE)

- Montreal Cognitive Assessment (MoCA)

Psycho-affective status

- Screening for presence of depressive symptoms (e.g. Geriatric Depression Scale)

- Structured psychological interview (for selected cases)

Nutritional status

- Dietary and nutritional history

- Standardized tests specific for nutritional status and generic for food knowledge

Physiotherapy

- Assessment of general motor capacity and of muscle-joint condition, balance, coordination, ability to perform the exercise movements and to use tools for the aerobic program

Management and self-management (individual and/or with caregiver)

- Adherence to drug therapy (by standardized test)

- Ability to self-manage drugs (using qualitative questionnaires)

- Self-monitoring capacity of weight and balance of liquids

- Self-control and management of hypertension and diabetes

- Symptoms recognition (by questionnaire)

V02, oxygen consumption. 
delivery of schemes that include times and methods for taking drugs, with the active involvement of the caregiver, especially when this person is also elderly, using appropriate communication methods that take into account the information needs and any cognitive, sensory, or socio-cultural limits.

Emotional, nutritional, and cognitive interventions. In the treatment of the elderly it is essential and indispensable, as already stated, to systematically evaluate with validated tools the cognitive status (its decline not always being clinically evident), and the presence of mood alterations, in particular depressive symptoms. Depression can in fact determine substantial changes in lifestyle or significantly affect both the functional prognosis and the risk of disease recurrence or death: these conditions can be addressed and treated with both psychotherapeutic and pharmacological interventions. Finally, the nutritional status requires professional assessment and intervention by the dietician, in order to identify the nutritional risk early, prevent and/or treat protein-energy malnutrition, and modify eventual inappropriate dietary habits with attention to sustainability by the patient and the ability to adhere to the prescribed diet.

\section{Future prospects}

The barriers to participation of the elderly in CR programs include: poor patient motivation, insufficient knowledge about its benefits on the part of doctors, the accessibility to facilities, and the lack of a geriatric culture in traditional cardiology to address major challenges (frailty, sarcopenia, physical deconditioning, cognitive and psychosocial aspects). It is necessary to identify the specific problems, the expectations and the preferences of the elderly heart patient, drawing up the general and specific goals to be pursued and using tools to detect these needs. Many elderly people who could benefit from CR programs have comorbidities that impact on mobility (e.g. dementia, osteoarthritis, peripheral neuropathy): it is therefore necessary to use specific methodologies for these patients, starting from a "multidomain" evaluation, followed by flexible individually-tailored interventions.

\section{CARDIAC PREVENTION AND REHABILITATION AS A MULTIDISCIPLINARY INTERVENTION}

\section{Minimum quality criteria for defining a multidisciplinary intervention of Cardiac Prevention and Rehabilitation}

In CPR different professionals perform, each with their own specific skills, the care activities that represent the cultural, organizational and scientific value of the discipline. Defining what is essential and indispensable to implement in clinical practice and its specific components in relation to the specific care setting is today of crucial importance because:

- there are no documents in this regard;

- the spectrum of patient profiles accessing CRP programs has broadened;

- different organizational conditions impact on the contents and modes for delivering the interventions;

- increasingly professionals have transversal functions in several services/hospital units.

GICR-IACPR recently set up a multidisciplinary working group for the drafting of Minimal Care paths to indicate those that, in the opinion of the Scientific Society, should constitute the minimum quality criteria for a multidisciplinary CPR intervention concerning the non-medical health professions (dietician, physiotherapist, nurse, psychologist). The ultimate goal is to avoid that the progressive reduction of resources available, organizational variability and the progressive increase in the complexity of patients lead to a situation of $a d$-hoc interventions dictated by contingency or by urgency and not the fruit of a coherent and strategic planning. The activity of the working group led to a publication indicated in the bibliography [20].

For each Professional Area, the specific objectives were identified: - Nurses: to identify and re-assess individual needs and cardiovascular risk factors. To plan and carry out interventions to meet the care needs in the different care settings, prevent risks related to the clinical condition and promote adherence to pharmacological (and non-pharmacological) therapy in collaboration with the caregiver. To program the aspects necessary for the continuation of care.

- Physiotherapists: to achieve the maximum possible recovery of autonomy and functional capacity compatible with the intervention time available and as tolerated by the patient. The initial assessment is critical for setting up a treatment aimed at the patient's real needs and appropriate to the care setting. Assessment during discharge is necessary to verify the results and plan the maintenance program.

- Dieticians: to assess/prevent/treat/monitor unhealthy eating habits to reduce obesity, dyslipidemia, arterial hypertension and diabetes mellitus. To satisfy nutritional needs in order to prevent or treat malnutrition and improve nutritional status.

- Psychologists: to evaluate/monitor the emotional state, the cognitive deficits detected, optimize the awareness/acceptance of the disease, identify and correct the behavioral and/or psycho-social risk factors, support the caregiver, promote therapeutic adherence, activate personal/socio-family resources and facilitate work, family and social reintegration.

Each intervention condition was analyzed considering:

- Biological factors specific to the disease (i.e. the "clinical" factors) and non-biological or "environmental" factors (family and economic situation, accessibility to care), and the interaction between them in delineating the type of patient and identifying the level of clinical complexity.

- Factors intrinsic to the specific expertise of each professional, to identify the different care complexity of the area.

We thus differentiated:

- A "clinical-assistance complexity", i.e. expressing the need for integrated multi-specialized and multi-interprofessional interventions. A patient is defined as "complex" based on different elements: consequences of the disease, coexistence of multiple morbid conditions, presence of complications, direct and side effects of the treatments carried out, cognitive deficits, disadvantaged social status, often with a further reduced organ/system function due to aging.

- An "Area complexity", definable as the condition in which a specific action is required on the patient who has a particular care need pertaining to the area of competence of that professional profile, regardless of whether or not the patient is the bearer of a high "clinical complexity".

Furthermore, the following were identified:

- Organizational factors such as the care context and the duration of the CPR intervention;

- Care context: outpatient $v$ inpatient;

- Duration of the CPR program: time is a fundamental variable for the health professional and for the patient, and the duration of the treatment can condition the goals and contents of the intervention, in particular for achieving results at the educational level for prevention.

The working definition of the Minimal Care paths was carried out by crossing the four dimensions: clinical-environmental complexity, com- 
plexity by professional area, care setting, and duration of the program, and analyzing the treatment goals, specific assessments and significant and essential interventions.

As an example, Table 10 presents the Minimal Care path for high clinical complexity. The pathways have been described in an analytical way, with a parallel representation of the interventions, but it should be emphasized that only an integrated approach can ensure an effective and efficient functioning of the multidisciplinary team as a whole. At the end of each pathway, all professionals must provide the patient and/or caregiver with access to information in order to make use of the various interventions available after discharge. The indications must also be prepared in writing and adapted to the patient's level of understanding; the goals pursued and results achieved, the individual/environmental resources/barriers identified and any problems still open or for which particular attention is required must be specified. In addition, the names of the team members must be reported.

\section{Attaining a sufficient level of clinical competence of the Cardiac Prevention and Rehabilitation team}

The level of professional experience of the interdisciplinary team in CPR is universally considered as a guarantee of intervention, i.e. as ensuring that the therapeutic goals are achieved and patients' quality of life is improved. At the same time, professional experience is both a structural and quality indicator of the Unit and of the CPR team as well as a requirement of the health worker for whom, probably, one of the main "business cards" is the possibility of exhibiting a core curriculum. The core curriculum is a "minimum curriculum" or "essential curriculum", i.e. the "minimum knowledge necessary" to be qualified as having the necessary knowledge and experience on the subject.

Regarding the physicians, the ESC finalized back in 2008 a "core curriculum for the general cardiologist" - a reference model for training, certification and continuing education in medicine - and the latest 2013 version [66] contains the following topics of potential interest for people working in CPR settings: 1) cardiovascular prevention, 2) physical activity and sport in primary and secondary prevention, 3 ) cardiac rehabilitation. In reading the original document, some passages can be extrapolated to help frame the profile of the "Prevention and Rehabilitation Cardiologist" and the relative professional competence. For example, at the point on prevention, the focus on the ability to manage non-pharmacological interventions, to promote global adherence to medications and lifestyle by coordinating and interacting with other health professionals, and the need to be an active part of community interventions: these are areas that are complementary to the canonical "generalist" interventions on the major risk factors (arterial hypertension, hypercholesterolemia, diabetes), which are often in daily practice limited simply to prescription and pharmacological review. Or, at the point on physical activity, the ability to utilize and modulate exercise as a drug in itself and as a vehicle for lifestyle change, over and above the ability to perform and interpret functional capacity assessment tests. Finally, at the point which really deals with rehabilitation, the capacity to grasp the individual patient's needs and be able to adapt the multidisciplinary and all-encompassing intervention to these needs, involving also the the caregivers in this and managing to set up a program for the long-term maintenance. These arguments would probably merit a contextualisation and further elaboration in the context of a "subspecialty curriculum" linked to CPR but unfortunately, in contrast to acute cardiovascular care, arrhythmology or imaging (https//www.escardio.org/Education/esc-and-subspecialty-core-curricula), this has not yet happened.

Even for those referred to by the unfortunate term "non-medical component" of the CPR, the same consideration probably applies as for the medical component, i.e. the absence of a defined core curriculum for those wishing to devote themselves to CPR - apart from more or less formal documents but these are almost always of a generalist nature promoted by the respective professional areas. In this sense, the identification of "what must be done in CPR always in the different areas", as enshrined for example by the recent Minimal Care paths of GICRIACPR [20], can be an excellent starting point for understanding the level of professionalism required to operate in a similar context.

Is it, in any case, possible to trace a basic training of the health professional in CPR integrating (but not substituting) the academic training of the individual figures (doctor, nurse, physiotherapist, psychologist, dietician, etc.)? A training syllabus aimed at constituting a common cultural terrain and therefore, ultimately, a strong interdisciplinary characterization? If so - as is desirable - who then should take care of this training, certifying the presence of a minimum common denominator of competence in the health professionals working in Italian CPR facilities? Obviously, the main actors in this process should be the institutions responsible for the task of Education (the Universities) and the bodies of representation and control of the Health Professions (the Orders). But also the Scientific Societies (in particular those of the sector) can play an active role and be themselves a place of cultural growth and constant monitoring of the situation and of the dynamics of "real life". Some practical notions could be the following:

To identify by consensus a single syllabus for the formation of the interdisciplinary team in CPR, defining the elementary teaching units with the relative levels of knowledge (superficial, general, detailed), competence (mnemonic, interpretative, decisional) and skills (theoretical, practical, autonomous) required. In the literature, some reflections have already matured in the field of cardiovascular prevention [67] and they may be useful to compile at least the main training blocks with the relative contents of this original syllabus (Table 11).

To assess systematically when mapping out the structural situation of the Italian CPR network (national census surveys such as the ISYDE series of the GICR-IACPR ${ }^{17}$ ), under the heading "organic characteristics", the degree of clinical competence of the members of the interdisciplinary team. This should be expressed not only by indicators such as length of service or the characteristics of the employment relationship (full- or part-time), but also by the presence or absence of certifications both individual and related to the structure as a whole. On this last point, for example, the possibility of accreditation by the European Association of Preventive Cardiology would seem about to be launched.

To promote, in agreement with the Academic world, post-graduate training courses taught by medical and non-medical health professionals with consolidated, recognized experience in the field of CPR, as was the case with the (1 1 st level) Master in Cardiac Prevention and Rehabilitation promoted by GICR-IACPR (https//spmsf.unipv.it/master/cardrp/index.html). Ideally, also in collaboration with the Regional Health Agencies, in order to incentivize and contribute to the training of health personnel working in CPR settings, as happened for example in the Sicily Region. This without forgetting also the "international" dimension of an adequate competence in the field of the CPR, which requires a global vision and acquaintance with non-Italian models, in order to organize and deliver an intervention in line with the epidemiological changes of the population of reference and with the evolving health systems; also in this field the establishment of partnerships for study or teaching mobility programs is of particular importance.

From now on, it is therefore highly desirable that in the agenda of each stakeholder interested in CPR (academic world, health control and accreditation bodies, scientific societies, hospitals and the single units or health professionals), the issue of clinical competence and of the relative obtainment and verification of it assume the status of a real priority. 
Table 10. High complexity Minimal Care path.

\begin{tabular}{|c|c|c|c|c|}
\hline & Nurses & Physiotherapists & Dieticians & Psychologists \\
\hline Goals & $\begin{array}{l}\text { Resolution of individual care } \\
\text { needs. } \\
\text { Knowledge about risk factors, } \\
\text { and behavioral changes } \\
\text { for prevention. } \\
\text { Promoting adherence } \\
\text { to treatments } \\
\text { Enhancement of } \\
\text { patient/caregiver resources. }\end{array}$ & $\begin{array}{l}\text { Recovery and improvement } \\
\text { of the level of functional } \\
\text { autonomy, respiratory } \\
\text { mechanics, joint mobility. } \\
\text { Increase of functional } \\
\text { capacity with aerobic } \\
\text { reconditioning. } \\
\text { Management of daily life } \\
\text { activities to prevent onset } \\
\text { of symptoms. }\end{array}$ & $\begin{array}{l}\text { Prevention and/or treatment } \\
\text { of malnutrition. } \\
\text { Reduction of excessive energy } \\
\text { intake, sodium and lipids. } \\
\text { Improvement of the quality } \\
\text { of lipids consumed. } \\
\text { Balanced intake of } \\
\text { carbohydrates and fiber. } \\
\text { Adequate dietary pattern (also } \\
\text { in relation to drug therapy). }\end{array}$ & $\begin{array}{l}\text { Promotion of motivation } \\
\text { to treatment and optimization } \\
\text { of disease management } \\
\text { at home. } \\
\text { Reduction of environmental } \\
\text { disorientation. } \\
\text { Active involvement of the } \\
\text { caregiver }\end{array}$ \\
\hline Assessment & $\begin{array}{l}\text { Care and educational needs. } \\
\text { Risk of infections, pressure } \\
\text { injuries and falls. } \\
\text { Detection of socio-familial } \\
\text { critical issues. } \\
\text { Verification of } \\
\text { self-monitoring capabilities. }\end{array}$ & $\begin{array}{l}\text { Degree of dependence } \\
\text { and needs for assistance. } \\
\text { Fatigue and dyspnea during } \\
\text { activity. } \\
\text { Autonomy and postural } \\
\text { transitions. } \\
\text { Balance: gait. } \\
\text { Falls risk. } \\
\text { Walk test, if possible } \\
\text { to perform. }\end{array}$ & $\begin{array}{l}\text { Nutritional risk. } \\
\text { Nutritional and dietary history: } \\
\text { energy and nutrient intake } \\
\text { (if intake is < } 50 \% \text { of resting } \\
\text { metabolism for } 5 \text { days or more, } \\
\text { evaluate indication for enteral } \\
\text { nutrition). } \\
\text { Anthropometric } \\
\text { measurements: weight, height, } \\
\text { body mass index, involuntary } \\
\text { loss of weight, } \\
\text { waist circumference. } \\
\text { Patient history - biochemical } \\
\text { examinations. } \\
\text { Physical examination in order } \\
\text { to evaluate nutritional status. } \\
\text { Food knowledge/skills. }\end{array}$ & $\begin{array}{l}\text { Presence/severity of anxious } \\
\text { depressive syndrome, self- } \\
\text { injury thoughts, psychomotor } \\
\text { agitation, refusal of self-care } \\
\text { and rehabilitative path, poor } \\
\text { family-member support. } \\
\text { Awareness about the disease, } \\
\text { motivation to treatment, } \\
\text { adherence. } \\
\text { Presence of cognitive } \\
\text { deficits. } \\
\text { Indication for referral } \\
\text { to Community services. }\end{array}$ \\
\hline Intervention & $\begin{array}{l}\text { Care plans/interventions } \\
\text { concerning mobility, risk } \\
\text { of falls and infections. } \\
\text { Education for symptoms } \\
\text { control, self-monitoring of } \\
\text { blood pressure, heart rate, } \\
\text { glycemic control, weight, } \\
\text { hydration and nutrition. } \\
\text { Instructions to } \\
\text { patient/caregiver for the } \\
\text { correct management } \\
\text { of drugs, implementation } \\
\text { of strategies based on } \\
\text { individual habits. } \\
\text { Individual/group motivational } \\
\text { counseling on the } \\
\text { management of risk factors. } \\
\text { Planning the aspects } \\
\text { necessary for carrying out the } \\
\text { treatment. }\end{array}$ & $\begin{array}{l}\text { Active-assisted mobilization } \\
\text { exercises. } \\
\text { Controlled breathing } \\
\text { exercises/pulmonary } \\
\text { reexpansion. } \\
\text { Segmental-postural gym } \\
\text { exercises. } \\
\text { Muscle strengthening } \\
\text { exercises for upper } \\
\text { and lower limbs. } \\
\text { Re-education in } \\
\text { walking/walking with aids. } \\
\text { Progressive training in } \\
\text { walking independently. }\end{array}$ & $\begin{array}{l}\text { Nutritional prescription: } \\
\text { - oral nutritional support } \\
\text { (high energy density foods, } \\
\text { modification of the } \\
\text { consistency of food and/or } \\
\text { drinks, oral nutritional } \\
\text { supplements) } \\
\text { - nutritional mixtures (mode } \\
\text { of assumption, monitoring } \\
\text { plan } \\
\text { - possible weaning from } \\
\text { artificial nutrition). } \\
\text { Nutritional education with } \\
\text { indications on the reduction of: } \\
\text { - energy intake } \\
\text { - sodium } \\
\text { - fats and their quality } \\
\text { (saturated, monounsaturated, } \\
\text { polyunsaturated and trans, } \\
\text { phytosterols and } \\
\text { cholesterol) } \\
\text { - alcohol and sugars. } \\
\text { Balanced intake of } \\
\text { carbohydrates and fiber. } \\
\text { Mode of food intake. } \\
\text { Food self-monitoring. } \\
\text { Group therapy educational } \\
\text { meeting for } \\
\text { patients/family/caregivers - } \\
\text { informational teaching material. }\end{array}$ & $\begin{array}{l}\text { Clinical interview for } \\
\text { evaluation and support } \\
\text { Possible psychometric } \\
\text { screening. } \\
\text { Relational strategies } \\
\text { to encourage participation } \\
\text { in the treatment course. } \\
\text { Environmental strategies } \\
\text { adapted to the residual } \\
\text { cognitive resources. } \\
\text { Support for participation } \\
\text { in psycho-educational group } \\
\text { interventions. } \\
\text { Motivational counseling, } \\
\text { activation of positive } \\
\text { affectivity and of } \\
\text { personal/socio-family } \\
\text { resources. } \\
\text { Facilitation of work, family } \\
\text { and social reintegration. }\end{array}$ \\
\hline
\end{tabular}




\section{The role of local organizations and patient associations}

In Italy, voluntary organizations, commonly known as the third sector, play a fundamental role of support to the institutions. Voluntary organizations are freely constituted organisms that operate on a nonprofit basis and perform activities of social solidarity integrating, or sometimes replacing, the public intervention if this is unable to give an adequate response to the problem in question.

The social and health sector is very developed in the field of voluntary activity. ISTAT data on "Voluntary organizations in Italy" in 2003 provide a snapshot showing the presence of approximately 21,000 Voluntary Associations; $28 \%$ of these deal with health care and $27.8 \%$ social assistance [68]. As in the past, still now, the Voluntary Associations are rooted above all in the Northern regions.

Right from the birth of the first CR facilities in Italy at the beginning of the ' 70 s, we witnessed almost simultaneously the emergence of the first associations of cardiac patients. These associations were often created as a spontaneous initiative of patients who had overcome an acute cardiac event, or sometimes on the initiative of cardiologists working in hospital facilities of acute Cardiology or CR. In any case, initially the main purpose for which these Associations were founded was to help support their members in the management of their cardiovascular problems in collaboration with the cardiological structures.
Over the years, and at the same time as the number of CR structures grew, these Associations have increased in number and membership. Some of these territorial associations of cardiac patients have linked together to increase their activity. In Triveneto, for example, since December 2000, the Triveneto Heart Federation has been joined by 28 Associations giving it a total of over 15,000 members (www.trivenetocuore.org).

In Italy there is also a National Coordination of Heart Associations (Conacuore) involved in the fight against cardiovascular diseases that currently unites together over 100 Associations in 18 regions (www.conacuore.it).

In the Statutes of these Associations, practically always the same goals are present, that define in practice the role they intend to perform and the objectives they aim to achieve. First of all, they aim to always operate in harmony and full cooperation with the Cardiology structures belonging to their territory, playing a complementary role and not one of subsidiarity.

The main scope of their operation is substantially to:

1. conduct activities of health information and raise public awareness about the various medical and social aspects of cardiovascular diseases;

2. promote initiatives, in collaboration in particular with the CR structures, in the fields of cardiovascular prevention and rehabilitation and health education;

Table 11. Proposed single syllabus for training of the interdisciplinary team in Cardiac Prevention and Rehabilitation.

\section{Training module}

1. Cardiovascular diseases

\section{Content of training}

1a. Pathophysiology of atherosclerosis

1b. Pathophysiology and clinical management of ischemic heart disease

1c. Pathophysiology and clinical management of heart failure

1d. Pathophysiology and clinical management of valvular heart disease

1e. Pathophysiology and clinical management of peripheral vascular disease

1f. Pathophysiology and clinical management of arrhythmic diseases

1g. Assessment of disability secondary to CVD

2. Estimation of cardiovascular risk

2a. Control of cardiovascular health

2b. Cardiovascular prevention strategies and guidelines

2c. Methods of estimating cardiovascular risk

2d. Cardiovascular risk communication

\section{Behavior modification}

3a. Psychosocial determinants of cardiovascular health

3b. Techniques for evaluating psychosocial and behavioral indicators

3c. Lifestyle modification techniques

4. Smoking cessation

4a. Pathophysiology of nicotine addiction

4b. Assessment of nicotine addiction

4c. Smoking cessation strategies

4d. Interventions on the general public

5. Nutrition and weight management

5a. Principles of nutrition and body weight management

5b. Nutritional and anthropometric evaluation

5c. Cardio protective dietary regimens

5d. Nutritional information and labeling

6. Physical activity and physical training

6a. Physical activity and physical training in cardiovascular prevention

6b. Principles of assessment of functional capacity

6c. Structured prescription of the individualized physical training program

6d. Population strategies

7. Risk factor management 7a. Hypertension

7b. Dyslipidemia

7c. Diabetes and metabolic syndrome

7d. Non-traditional risk factors

7e. Evaluation and ways to combat non-adherence 
3. economically support, when necessary and possible, the needs of the reference cardiac facilities, in particular as regards the renewal of the technological equipment necessary for daily activities;

4. support the cardiological research activity.

The tools with which to achieve these objectives are essentially the following:

1. organization of periodic meetings to inform their members and the general population on specific cardiovascular issues;

2. collaboration with schools, especially primary schools, for meetings with students on health education issues to raise awareness about correct lifestyles and aimed at the primary prevention of cardiovascular diseases;

3. organization of courses of maintenance gymnastics for chronic heart disease patients to be carried out in dedicated gyms with the aid of properly trained physiotherapists and with supervision and advice from expert cardiologists of physical training;

4. organization of collective physical activities such as "walking groups";

5. organization of convivial meetings and group trips for socialization purposes;

6. implementation of awareness-raising days for the general public in which voluntary contributions dedicated to the development of specific initiatives can also be requested;

7. organization of days in which the general population is invited to undergo a free check-up of the main cardiovascular risk factors with a risk card compiled and delivered, by the cardiologist present, to each screening participant during a final interview;

8. implementation every year of the World Heart Day;

9. publication and free distribution of periodicals and leaflets of medical information on cardiovascular diseases.

All these types of initiatives must obviously be carried out in close collaboration and with the help of the Cardiology of reference.

The initiatives promoted by the various local associations of the Friends of the Heart to spread the culture of cardiovascular prevention among the general public are always useful, but they are certainly even more effective when carried out in a coordinated way and pursuing common specific objectives.

For this reason, it could be very useful that the Associations not yet linked with Conacuore adhere to this national coordination born in the late 90 s with the primary scope of reuniting the various local associations of Friends of the Heart, while maintaining their total organizational autonomy and decision-making, to develop common projects and intervention strategies and coordinate the various activities carried out in the area.

Finally, it is very important that patients' Associations become increasingly spokespersons vis-à-vis the Institutions of the importance of CPR, access to which is still too often not assured to many cardiac patients, with increasingly evident consequences on the health of Italian citizens and healthcare in the western world.

\section{How to harmonize the diagnostic-therapeutic care pathways of Cardiac Prevention and Rehabilitation with the different regional norms}

The diagnostic-therapeutic care pathway (DTCP) is a method to manage the global course of care and assistance of a patient with a specific clinical problem. It involves interventions, more or less complex, characterized by the preparation and implementation of predefined activities usually carried out by multidisciplinary teams. These represent basically an elective instrument for the control of a specific pathology or particular clinical problem delineating the best practicable path within the network in which it is inserted.
The elaboration of a DTCP aimed at the delivery of a specific medical intervention must therefore take into account the best evidence from the literature, substantially represented by national and international guidelines and recommendations, but also national and regional legislative provisions concerning the organizational models. Always naturally respecting the Essential Levels of Care.

The elaboration of a DTCP, in addition to listing the priority health skills, involves also expertise of the economic-administrative sphere to assess the impact of the DTCP from the point of view of the budget available and economic sustainability. And it is not rare to see, in the elaboration of the DTCP, a divergence between what it would be necessary to provide in order to have the best possible quality of care and how much it is practically possible to provide. Each DTCP must ultimately be contextualized in the everyday reality so that the path prepared represents the best possible route in that given organizational and functional situation.

Also in CPR, clinical-organizational pathways have been designed, in particular by the Scientific Societies of the sector. Specifically, scientific documents are available that define with great clarity and intellectual honesty, based on well-documented literature data, the appropriate clinical criteria for access to CR interventions, the criteria for priority of access to rehabilitation (taking account of the current impossibility to offer everyone what would be clinically necessary), the appropriate contexts for the provision of rehabilitative intervention (interventions in inpatient or day-hospital regime, simple or complex outpatient procedures) and the structural-technological-organizational requirements necessary to provide a qualitatively adequate intervention $[2,26,40,41]$.

Effective and efficient courses have been developed that also consider aspects that are not directly clinical, with the aim to obtain the best possible result for the patient with the most appropriate use of available resources.

On the institutional and legislative side, there is also an abundant literature concerning rehabilitative activities starting from the "Guidelines of the Ministry of Health for rehabilitation activities" published in the Official Gazette on May 30, 1998 up to the Rehabilitation Plan of the Ministry of Health of 2011 [39,69].

In the years since, the regionalization process has led to the transfer to the periphery of many legislative functions, giving each region wide autonomy especially in the field of health management. Hence, in the face of more or less generic Proceedings issued by the Ministry of Health each Region has dealt independently with the health interventions, with often contradictory results between one region and the next.

In some cases, Regions have acted in a propositive and positive manner in the last few years, interfacing with specific health skills in order to build shared and appropriate management models. In other cases, also due to the fact that the legislation makes each Region responsible for covering its own eventual financial deficits, the Regional Resolutions, in particular in those Regions subject to budget spending restrictions, seem to have as their sole purpose and objective to reduce to a minimum any form of rehabilitation intervention, regardless of the documented clinical need, not unlike what is proposed at the level of the Ministry of Health, some AIFA Notes and some Therapeutic Plans for the implementation of innovative drugs.

Not infrequently, moreover, these institutional documents have addressed rehabilitation in cardiology and not CR or CPR, thus linking the intervention of CR with that of physiatric or neuromotor rehabilitation, treating CR in a generic way and without specific connotations, starting from the question of what specialist should be responsible for the rehabilitation project/program (not in all regions is it the cardiologist) or what specialization the Director of a Simple or Complex Unit of CR should have. 
To illustrate some modes of acting and compare the norms present in the various Regions we outline here below the situation of some important Italian regions. For needs of synthesis, we will not proceed to an analytical examination of the different experiences, but will limit ourselves to highlighting those aspects of particular importance, leaving a deeper analysis up to the reader.

\section{Lombardy region}

In this Region, Technical Tables were set up by the Health Directorate in which the members of the Cardiac Rehabilitation Subcommittee of the Cardiocerebrovascular Commission took part. These led to several resolutions on the reorganization of rehabilitation activities, the appropriateness of inpatient rehabilitation and in particular, in 2009, a Decree of the Directorate General for Health on the "Appropriateness of the activities of Cardiac Rehabilitation in the Health System of the Lombardy Region". In this Decree, many of the indications of our Scientific Society were incorporated (amongst which, a clear statement that "the responsibility of the Operative Units of Specialized Cardiac Rehabilitation should be entrusted to a cardiologist specialist") [70]. Nevertheless, the regulations still in force in the Lombardy Region date back to 2004 [71]. This legislation has many limitations: in particular, it considers cardiac and respiratory rehabilitation entirely ancillary to physiatric and neuromotor rehabilitation, on whose needs the legislation was based; rehabilitation in cardiology (and not CR) was therefore treated in a nonspecific manner and the figure of the cardiologist totally marginalized to the point of declaring that the presence of a cardiologist in the team was necessary only when at least $50 \%$ of the patients were cases attributable to CVD [5]. A reform of this legislation, launched in 2014 and which ought to resolve many of the critical issues of 2004, has not yet been completed.

\section{Veneto region}

Also this Region, which back in 2007 regulated with a specific Regional Resolution the appropriateness of admissions to Rehabilitation Units, in recent years has seen an active collaboration between regional operators and physicians working in the various sectors of rehabilitation, taking into account the numerous innovations in the field. At the end of a journey lasting more than 2 years and characterized by the implementation of numerous technical-scientific meetings in Technical Tables set up by the Region, a Declaration was published in July 2016 by the Region in which, in a specific Annex, precise indications were issued regarding rehabilitation activities [72].

In the specific case of $\mathrm{CR}$, precise indications were given on the criteria of clinical appropriateness and rehabilitation access, even if the assessments were not always completely shared, especially as regards rehabilitation interventions distant in time from the index event.

\section{Sicily region}

In this Region an important collaboration in Technical Tables set up by the Health Department between rehabilitative physicians and regional officials led to the publication in the Official Gazette of the Region of Sicily of the "Rehabilitation Plan" [73]. Within the Plan, in a specific annex, the Plan of Cardiac Rehabilitation in Sicily appears as a consensus document, elaborated by the working group in which Sicilian rehabilitation cardiologists participated.

Very specific clinical-organizational rehabilitative pathways are developed in a very detailed way, real rehabilitation DTCPs. Moreover, in the document, in consideration of the complexity of the cardiac rehabilitation intervention, the need is recognized for a cardiologist specialist to be assigned the responsibility of the rehabilitation facilities and also that the admissions to CR have a specific 56 code: 56.08 . Re- garding this last point, which has a rational and indisputable logic, so far in no other region has a similar proposal been formulated.

In conclusion, in many Italian regions much work still needs to be done to build an adequate and appropriate rehabilitation offer. Only through a real and collaborative confrontation between rehabilitation cardiologists and ministerial and regional technicians will it be possible to elaborate efficient rehabilitation pathways and therefore offer the patients who need it an adequate intervention within the National Health System.

In many regions to date these interventions are still below the minimum standards, although there are many data that certify that, within the various rehabilitation pathways, the cardiac one appears to be the most appropriate in terms of the clinical organization and delivery.

In particular, the institutional document of the Ministry of Health of October 2013 "Methodology for the definition of criteria/parameters of appropriateness and efficiency of hospital rehabilitation admissions" clearly shows that of the various types of rehabilitation intervention (neurological, orthopedic, respiratory and cardiac) the cardiological one is by far the most appropriate and correct [74].

\section{Unmet clinical needs: Increasing the rate of referral to Cardiac Prevention and Rehabilitation}

Current rates of referral to structured CPR programs in patients with cardiovascular disease - generally not more than 20\% [75] - are certainly unsatisfactory and deprive large portions of the population of an extremely effective intervention in terms of reducing the related disability, morbidity and long-term mortality. The Italian National Health System recognizes CPR activities within the framework of Essential Care Levels, albeit with inter-regional differences regarding the norms of appropriateness and provision of the intervention, placing itself thus outside the large group of nations (even with advanced health systems) in which the lack of reimbursement of benefits constitutes the main barrier to access. Nevertheless, even in Italy, the referral rate remains low (estimated at around 30\%), regardless of any other assessment of the degree of response to the demand or of the performance of the rehabilitation network.

The low referral rate needs to be framed within the broader perspective of recognized conditions underlying the non-participation (and frequent interruption) in CPR programs. These conditions have recently been the subject of a systematic review [76] and grouped into six main categories: intrapersonal factors, clinical factors, interpersonal factors, logistic factors, factors related to the CPR program and factors related to the health system. As shown in Table 12, the lack of referral - if compared to other factors of considerable importance - is the main cause of exclusion of patients from a rehabilitation program; in other words, the request for CPR cannot be completely expressed autonomously by the patient and still requires the strong mediation of a health proessional - in the hospital or local community - appropriately competent and sensitized.

The first answer to the question "how to increase the rate of referral to CPR?" could therefore simply be to: "remind the potentially referring physician of a care obligation and that renouncing to indicate CPR should be seen in the same light as renouncing antihypertensive therapy in the hypertensive patient, or a statin in the hypercholesterolemic patient, or a stent in an infarct patient with surgically viable coronary arteries". That is to say, as an exception and not the rule and, in any case, as a poor professional performance.

From a more detailed perspective, further actions that can be proposed are:

1. For those involved in the field of CPR, to carry out a finer-grained analysis of the phenomenon of referrals, giving our Scientific Society in the sector a greater role in organizing and conducting na- 
tion-wide surveys and polls, searching institutional databases, interfacing with the representatives of acute cardiologists and of GPs, seeking to combine both the macro and the micro dimension (from national trends to the smaller local realities). In fact, there are different ways of understanding the referral rate: e.g. as the number of patients referred to CPR in rapport to the total number of potentially eligible patients and for whom referral was indicated, regardless of who gave the indication; or, as the number of patients referred to CPR by a given provider compared to the number of potentially eligible patients observed by that provider; or again, as the number of providers that, evaluating a given patient, indicated referral to CPR compared to the total number of providers that evaluated the patient. The use of different observation windows would therefore allow to evaluate (and ideally influence) multiple dynamics related to conviction, tradition/custom, inertia, tendency to innovation, and the presence of biases on the part of providers who are potential "commissioners" of CPR services for their patients. Finally, we specify that the term "provider" refers to both individual physicians and work groups (e.g. staff members of acute wards or GP consortia).
2. To organize at national level a process of accreditation of structures and professionals (especially those in a managerial position) that deal with CPR.

3. To promote the "Rehabilitation Facility Plans" (RFP) of CPR structures according to the definition of the Rehabilitation Plan of the Ministry of Health [77], i.e. instruments with which each structure defines ex ante its characteristics, the types of services offered, the work potential and requirements, the professionals involved in the team and their specific skills, the admission/discharge procedures and relations with other structures. The greater availability and visibility of well-constructed and appropriately disseminated RFPs will in fact be able to provide adequate information to those potentially interested, facilitating a proactive attitude for referring patients. The latter, in particular, appears to be an ambitious goal, since it is not easy in the healthcare world to convince health operators to make public the precise organization of their work, due to the fear of emergence of weak points and hasty labeling. On the contrary, the growth in our network of open-minded CPR structures would lead not only to an easier acquisition of good clinical practice, but could also raise the level of priority of CPR in the eyes of the potential providers, particularly as regards acute care.

Table 12. Factors associated with lack of patient participation in the Cardiac Prevention and Rehabilitation (CPR) program and the relative risk (expressed as odds ratios), classified by macro-categories.

\begin{tabular}{|c|c|}
\hline \multirow{2}{*}{$\begin{array}{l}\text { Factor } \\
\text { Intrapersonal factors }\end{array}$} & \multirow[t]{2}{*}{ Odds ratio } \\
\hline & \\
\hline Elderly age & $0-58-4.76$ \\
\hline Female sex & $1.64-4.17$ \\
\hline Low education level & $1.5-1.81$ \\
\hline Low income level & $1.47-5$ \\
\hline Depression & $1.20-3.85$ \\
\hline Low self-efficacy & $1.05-2.17$ \\
\hline Reduced awareness of the benefits of rehabilitation & 10.11 \\
\hline Reduced motivation & $1.04-17.32$ \\
\hline \multicolumn{2}{|l|}{ Clinical factors } \\
\hline Smoking & 1.69 \\
\hline Reduced functional capacity & 1.13 \\
\hline Hypercholesterolemia & $1.82-2.73$ \\
\hline Hypertension & 1.72 \\
\hline Diabetes & 1.82 \\
\hline Peripheral vascular disease & 2.32 \\
\hline Heart failure & 1.28 \\
\hline Previous coronary revascularization & $1.56-1.82$ \\
\hline \multicolumn{2}{|l|}{ Interpersonal factors } \\
\hline Absence of partner & $1.30-16.73$ \\
\hline Reduced social support & $1.12-1.22$ \\
\hline Unemployment or retirement & $0.48-5$ \\
\hline \multicolumn{2}{|l|}{ Logistic factors } \\
\hline Distance from the rehabilitation facility & $1.16-10$ \\
\hline Dependence on transport & 3.09 \\
\hline Lack of transport & 1.85 \\
\hline Rural area & $1.91-4.55$ \\
\hline \multicolumn{2}{|l|}{ Factors related to the CPR program } \\
\hline Previous participation & 0.42 \\
\hline \multicolumn{2}{|l|}{ Factors related to the health system } \\
\hline Lack of referral & $4.03-2514$ \\
\hline Insufficient intensity of recommendation & $1.49-2.04$ \\
\hline Referral to a public structure & 1.54 \\
\hline
\end{tabular}


4. To optimize collaboration with Patient Associations to foster awareness-raising among patients themselves and of public opinion about the importance and health outcomes of CPR, as an aspect of the patient's care pathway that needs to be assured. As a final note, it is important to reiterate that there are no universally applicable recipes to counteract the phenomenon of the limited rate of referral to CPR. The fact that this is probably a global phenomenon and that a greater demand for CPR services would in turn generate the problem of how to fully satisfy such a demand are not, however, valid reasons for not implementing corrective strategies.

\section{From current medicine to Precision Medicine: Where Cardiac Prevention and Rehabilitation stands}

Someone has defined current standard medicine (based on evidence) as a "Medicine of Imprecision", classifying the best that can be done today as "Individualized Medicine" while the type of medicine we are trying to build, hence our objective, is "Individual Medicine or Precision Medicine" [78]. As is known, evidence-based medicine is based, when possible, on randomized controlled clinical trials. The results are often generalized and become prescriptive. In reality, each person has their own unique genotype and phenotype and any "generalizable" knowledge has limits. The benefits or negative results of evidencebased medicine apply "to the majority" and, among this majority, to some more than to others. Individualized Medicine, on the other hand, imposes a precise characterization of the phenotype as its platform. Characterization includes all the known factors capable of modifying the clinical expression and effects of therapies: gender, age, clinical profile, comorbidities (essential), information derived from instrumental technology, biomarkers, response to drugs, cognitive status, environmental/social situation, etc. In other words, it means to best use what is available to characterize a patient before making a diagnosis (as specific as possible) and consequently a therapy (if any) that has an appropriate rationale. Precision Medicine is what is in the course of being configured with the growing technology applicable in everyday life, the pool of shared observational experience (registers, electronic health recording, smartphones), used continuously to test and define preventive, diagnostic and therapeutic paths, welding together pathophysiological research and clinical research. Today's evolved medicine is moving from the first to the second stage, the third is completely experimental. Some aspects can be relevant in this process also for CPR.

A first new, decisive aspect for all involved in chronic disease pathways, first and foremost CPR, will be the technical and cultural capacity to manage medical-scientific relationships over time, even at a distance. This, thanks to the use of appropriate technologies (wireless sensors, telemetries, long-term outpatient recordings), prompt reaction with the various means of communication - current and future even in trivial contacts with patients or those involved in prevention programs, the immediate availability of personal historical data, and a network of links that can quickly resolve the patient's needs, through activities if not available within the facility itself available in pre-established collaborative networks. This represents an important prospect for CPR: to be a vital and stable (because useful) segment in patient pathways using all the rapidly evolving technology available, and hence being present in the daily medicine network.

A second aspect is the social role that CPR is assuming in the technically advanced world, particularly in Europe and North America. The ESC has clearly defined its roles and working procedures [55]. Naturally, since the ESC is a Federation of many countries [79], it formulates standard operational working recommendations that then need to be adapted to the structurally very different realities. Moreover, the European Union does not issue guidelines, which might in some way have coercive implications, not always welcome, because the countries that make up the European Union have full independence in health matters.

The situation is different in the United States where the healthcare system has an important common, federal identify [24]. The U.S. is working intensively on some key aspects of public health. First of all, the implementation of the "Electronic Health Recording" system, consisting of a nation-wide computer network for the systematic collection of health data produced during daily activities (i.e. observational). The data collected are ordered into clinical, administrative, management, technological sub-areas, etc., and (in theory) should be fluidly interoperable. In practice, it will constitute a comprehensive and dynamic analytical observatory of the current health world [80-82].

What is essential is that, in the U.S., they intuited from the beginning that the system had to incorporate and always be guided and verified by scientific research. To date, over $\$ 30$ billion has been provided to the National Institute of Health for research into the production and quality of scientific data related to Electronic Health Recording [83]. In parallel with the path of observational research, a strong line of research in international medical statistics is being developed to suggest new approaches both in study designs and in data analysis in order to extract relatively solid information even from terrains that are notoriously prohibited for observational research, e.g. causal links between treatments and clinical events (properly studied through randomized trials). In addition, American medical-scientific societies produce very practical working guidelines [25]. A recent, fundamental, document of the American College of Cardiology and of the American Heart Association, concerns the clinical performance and quality measures of Cardiac Rehabilitation and clearly establishes the various operational steps of a rehabilitation process [25]. The Task Force that drafted the document has maintained close relationships with users, represented by U.S. public medicine, in particular the Centers for Medicare and Medicaid Services (CMS).

The candidates for rehabilitative care - practically all patients with acute and chronic ischemic heart disease and patients with heart failure - are defined strictly on the basis of the published data, and the tasks of the cardiologist are specified in carrying out the activities. The current situation is discomforting: today only one-third of the patients are referred to rehabilitation and only half of these actually complete the program [25]. But now, in agreement with the CMS, the situation is destined to change. The hospital doctor in charge of a patient without specific contraindications to rehabilitation (physical or otherwise) is required to offer the patient this opportunity and to note the patient's response in the clinical record. If this does not happen, both the doctor and the hospital will acquire a point of demerit [25]. Considering that merit and demerit points are public and translate, respectively, into incentives or sanctions, this may not be irrelevant. With this operative insertion pre-concorded with the public health system (CMS) and determined by the federal administration of the Health System, CPR has now entered to become a component part of the cardiovascular pathways in the US Health System.

The third aspect, very important, is contained in another recent document, also from the United States, in which a concrete and prospective picture of Precision Medicine is outlined. The title is explicit: "Statement on Healthcare Transformation in the Age of Digital Health, Big Data and Precision Health". The paper was written by a Task Force on Health Policy Statements and Systems of Care of the American College of Cardiology [84]. It proposes a comprehensive and interactive model of current technological and intellectual forces, and is aimed at research and clinical practice. It is schematically shown in Figure 2. It should be underlined that it is not a figured and colored list of potential data sources, but a synoptic framework based on current scien- 
tific knowledge without ever losing sight of the end user, the patient. The technical platform, the collector of data - Digital Health - highlights in particular the increasing multiplicity of portable sensors of biological signals in normal life and their remote monitoring, i.e. a fundamental observational penetration of medicine (or, more generally, of the biological pathophysiology of the human body) into the daily life of the human community.

An important element, as a future prospect, is also the so-called "Artificial Intelligence". The term might be "unfriendly" or at least intriguing. Conceptually, the topic has clear objectives. More or less it is about imitating human intelligence with computer systems. Technically, this is done by setting up computer learning techniques that imitate the processing and memorization of the human brain (machine-learning techniques, learning machines), arriving at deep learning techniques, algorithms that should solve clinical problems (but, obviously, not only). Needless to mention here the gigantic technical and, above all, ethical-social problems that will arise. Another key element of the document concerns the collection and management of data (Big Data). It focuses on the spread of Electronic Health Recording, but above all on the universal use of smartphones (used today by more than 3 billion citizens), on the increasingly frequent access of people to sources of health information (over 160,000 Health Apps on the market) and the willingness of users to provide data that concerns them in the long run. Finally, attention is placed on Precision Health, which focuses on the aggregability and traceability of data of each individual coming from different sources that concern genetics, pathophysiology, biomarkers, socio-environmental factors and other areas that provide phenotypically characterizing elements for each subject.

This conceptual and operational framework is, for now, a prospect, an anchor of rationality to complete the technological tools considered and make the end realistic - public health as a primary asset - today not pursued in many countries. It will take time to consolidate as an operational strategy [85]. However, CPR could adapt itself more or less to this scheme, portraying an image of itself as an open medical area, well-educated, sufficiently technologized to provide etiopathogenetic insights, able to accompany patients (who will increasingly determine the social role of medicine) with a participatory and highly professional approach, offering continuous availability over time, combined with the confidence to obtain what medicine can give. Along these lines, in the United States, large collaborative networks are already moving. Amongst these is the Million Hearts Cardiac Rehabilitation Collaborative, which aims to participate in the CR programs of $70 \%$ of the eligible CR candidates by 2022 , and estimates the prevention of 25,000 deaths and 180,000 hospitalizations per year [86]. An ambitious result, but one that could also be vastly enriched by using other parameters of reference, for example the complex and increasingly valued area of quality of life.

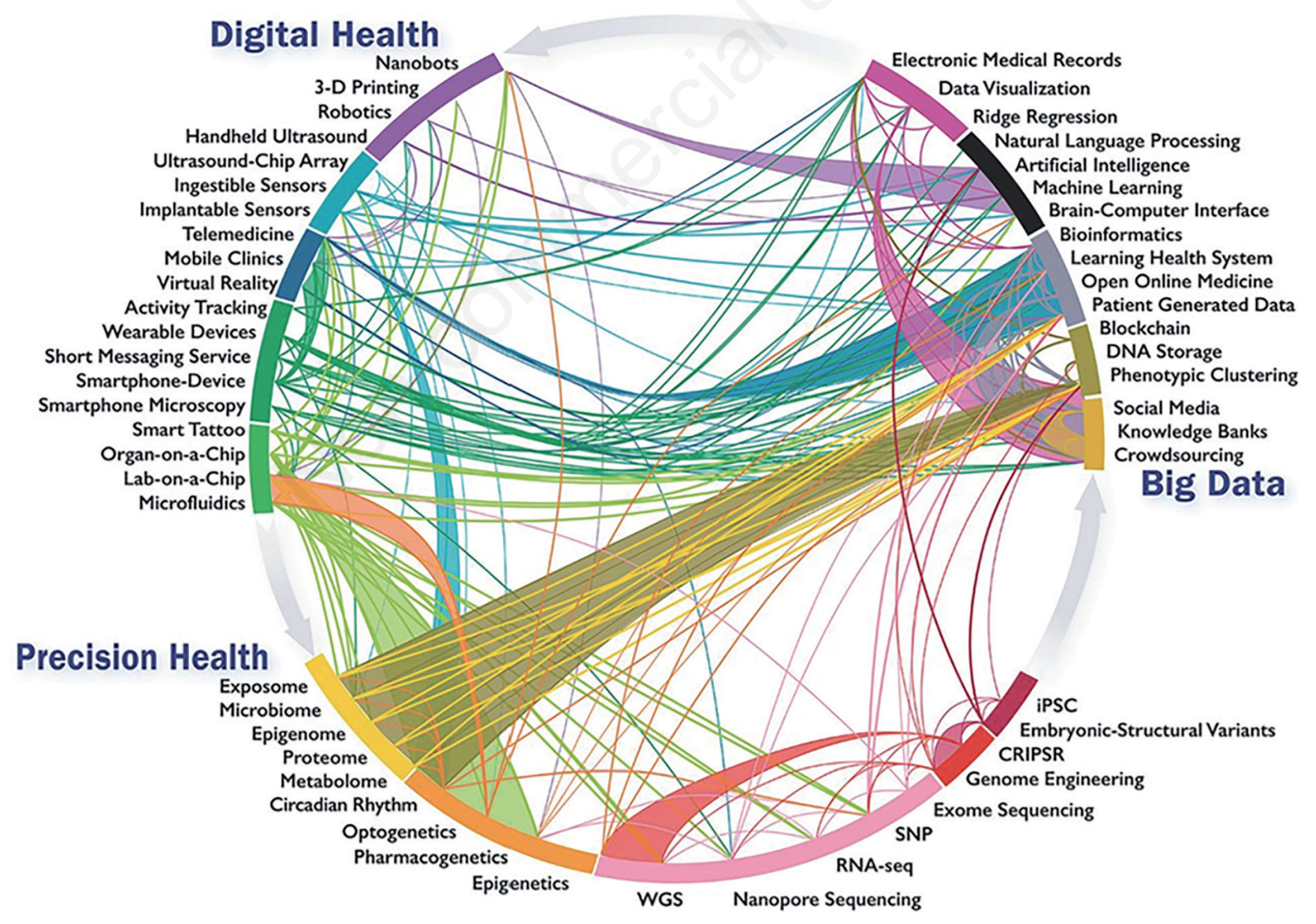

Figure 2. Scheme of innovative components in medicine and their intra- and interconnections [62]. Reproduced with permission from Bhavnani et al. J Am Coll Cardiol 2017;70:2697-718 [84]. 


\section{Innovation and research in Cardiac Prevention and Rehabilitation}

It may seem paradoxical but at the end of 2018 there still is need to dispel doubts about the effectiveness of CPR. There is a certain skepticism about the need for a CPR intervention due to the advanced degree of development achieved by modern cardiological therapies as regards drugs, reperfusion strategies and devices. After the publication of the "negationist" RAMIT trial [87]- which was conducted in 2012 and disproved the positive effect of CPR in terms of mortality, morbidity, modification of risk factors, functional capacity and quality of life after myocardial infarction - in the following years there was a succession of pros and cons by means of reviews and meta-analyses, which had as object not only the technical-statistical aspects of the study. The main merit of this diatribe, in fact, was to highlight the importance of how the rehabilitation program is structured, as an aspect that can strongly influence the results of the program itself. In other words, not everything can be passed under the term of CPR, and less structured interventions with a high rate of interruption (as in the case of RAMIT) present a high risk of failure, with negative consequences not only for the individual patient but also for the health system in which the intervention has taken place.

On this fundamental theme, modern research must continue to be committed, despite the significant obstacle due to the impossibility for obvious ethical reasons - of organizing randomized clinical trials with control groups excluded from a CPR intervention in the fields of both ischemic heart disease and heart failure, in consideration of its highest Class I recommendation with level of evidence A. Innovation and research in this field therefore probably go hand in hand, not so much in the elaboration of finer abstractions from previous studies, but in the preparation and verification in the field of working protocols, hopefully also with the use of modern instruments of e-Health.

Then there are specific aspects - weak points generally - that require further clinical research effort (also here of a combined observational and operational type) to generate corrective measures and positively affect real life. Firstly, the issues of the reduced rate of referral to CPR, the barriers to access, and the low adherence to the program itself. In this sense, it is gratifying that an all-Italian multi-center study such as GOSPEL is still regarded as a reference not only in terms of the results obtained by the program, but also in terms of the methodology used [10]. Secondly, the identification of special programs for selected patient populations, e.g. frail patients and the female gender, definitely stands out. Furthermore, the development of alternative models to bridge the gap between the demand and supply of CPR services, particularly at the community level, or utilizing new technologies to increase patient self-efficacy.

Another issue that is certainly fascinating from the cultural point of view is that of epigenetics applied to CPR, that is the study of the different gene expressions in response to rehabilitation protocols, e.g. in the field of physical training or dietary modification. On this subject we are still at the dawn of research. However, we can glimpse the enormous potential for which it will be possible, in the future, to calibrate the rehabilitation program not only on cardiovascular risk scores or disability scores derived from population studies, but also on the patient's individual phenotype and to be able to induce, thanks to the CPR intervention, epimutations positive for cardiorespiratory and muscle function.

From a very practical point of view and oriented towards providing decisional support to the health professional in CPR, it would also seem important to promote and incentivize the research already in progress [88] concerning the harmonization between DTCPs, identification/qualification of rehabilitation needs based on ICF (International Classification of Functioning), elaboration of the Individualized Rehabilitation Plan, and selection of components of the individualized rehabilitation program. A harmonization that requires further contextualisation not only in terms of the different groups of access to CPR, but also of the different care settings, with the appropriate modulations required for the inpatient $v s$ outpatient setting. On this point, we can also see huge benefits with regard to the future digitalization of all these processes, which will have undeniable potential gains in terms of the working mode and reappraisal of the multi-interdisciplinary character of the intervention.

In some respects, innovation in CPR would also mean to rediscover some historical roots of this discipline. For example, by reaffirming in the healthcare community the wealth of expertise in the area of functional assessment of cardiac patients and prescription/administration of a highly structured physical training intervention, or in particularly complex patients. A recent multicentric observational survey of GICRIACPR [89] in fact highlighted - even in centers of CPR with a solid tradition and a high number of patients - very low rates of performance of advanced functional tests (such as the cardiopulmonary test) or complex training tests (multidimensional or high-intensity exercise tests), generally not more than $20 \%$. This phenomenon can be attributed at least in part to the characteristics of greater instability and frailty and to the shorter time interval after the acute event of the referred patients. However, the identification of "modern" strategies to ensure in any case a fine structured assessment of functional capacity even in these patients - with all the benefits that result in terms of prognostic stratification, implementation of a personalized training program and desirable benefits on the activities of daily life [65]- could be a reasonable goal of current research.

Finally, from a national point of view, the need is felt by many for research that is "convergent" with the main lines of the current healthcare reforms, which in some Regions have seen substantial changes in the provision of services and players involved, developing collaborations with and analyses of administrative databases. Doing research and innovation in CPR in this sense would mean: 1) collocating CPR in the treatment pathway of acute and chronic heart disease in each regional health system, clearly defining the relationships with the stakeholders upstream (the sphere of acute care) and downstream (local community care); 2) evaluating the overall performance of CPR and directing its operation based not only on of a concept of priorities, but also on cost-effectiveness; 3) opening CPR up to systematic analysis, borrowing some methodologies from the economic sciences; 4) accepting elements of rationalization and remodulation, knowing how to evaluate the clinical-organizational repercussions with scientific method and formulating suitable working proposals in support of the institutional bodies.

Finally, as described in the previous section, CPR can effectively take a leading role in the research scenario concerning the profound and tumultuous process of change taking place in Medicine with the interaction between Digital Health, Big Data and Precision Health.

These and many other topics could be put on the agenda to promote research and innovation in CPR.

\section{CARDIAC PREVENTION AND REHABILITATION IN 2018: SHADOWS, LIGHTS, PROSPECTS AND NEEDS}

The use of a software numbering in the title of this position paper is not accidental - it was done to indicate the current positioning of CPR in a path that began back in the distant past. Why in fact talk about "CPR 3.0"? Because over the course of the decades we have witnessed 
a progressive and continuous evolution that has seen our discipline change, from being almost exclusively "exercise-centric, version 1.0", to then go on to become "multidisciplinary, version 2.0", including also activities of prognostic stratification, clinical stabilization and optimization of therapy. In the current historical phase, characterized by an epidemiological evolution that has led to a marked increase in the elderly population - destined to continue - with an equally important growth of chronic pathologies, it is important that CPR also conducts a critical review and undergoes a process of adaptation to this new reality. Hence the reason for a "version 3.0".

The contribution that we want to make here in this section is to underline the current situation, with its shadows and lights, and to spell out the future prospects/needs, drawing on what has been reported in the position paper as a whole.

\section{Shadows}

1. CPR, despite its decades of history and the great vitality demonstrated both at a cultural and operational level over the years, continues to be not adequately considered within the cardiology scene. Furthermore, the importance of CPR at the clinical level and the "health" benefits it provides are not well known by the beneficiaries themselves, i.e. patients on the one hand and the health system on the other. There is probably still some prejudice against CPR based on fundamental misunderstandings: perhaps it is thought that prevention is something that all health professionals already know and realize, or that rehabilitation is nothing but physical activity, not much different from what can be performed in a gym, simply with greater health supervision. As far as the health system is concerned, there continues to persist a vision of a close association between rehabilitation and physiotherapy, a vision inherited from neuromotor rehabilitation (probably no longer adequate even for the latter) and translated sic et simpliciter to cardiac and pulmonary rehabilitation. This seems to persist despite innumerable consensus documents elaborated by the various Scientific Societies that, linked together on the cultural level, show that their recommendations lie extremely far from this "base".

2. The only concept that seems to have made a breakthrough in daily clinical practice is that of considering CR as useful for "freeing up beds" in acute care hospitals. This aspect is placed between the "shadows" not because it is in itself negative but because CPR cannot be interpreted as "a discharge rehabilitation". If this were so, it would soon become little different from long-term care, a lower-cost care setting where to refer the acute patient, with problems more prevalently social than health, and not a field of care with different settings and different levels of treatment intensity, to which patients must be initiated according to their needs and their actual chances of recovery.

3. The fact that the so-called "rehabilitation in cardiology" is considered by the health care system and by the institutional interlocutor, as a part of rehabilitation and not of cardiology. This leads to a loss of specificity and characterization, not useful for CPR and especially for patients.

4. The reality of CPR in the national territory is extremely varied, both from the point of view of its actual presence and the type of services offered. The CPR offer is also biased towards the inpatient regimen compared to the outpatient and often does not reflect the organizational and functional characteristics necessary to guarantee an adequate treatment outcome.

5. Even if this position paper has referred to the so-called "secondary prevention clinics" several times, the feeling is that in reality we are talking about a very fluid entity. Over and above the outpatient pathways, simple or complex, which are attached to inpatient CR facilities or classify themselves as outpatient rehabilitation facilities, most of these "secondary prevention clinics" coincide with the outpatient clinics for "ischemic heart disease or post-infarct" of acute care hospitals. And even if they carry out a fundamental and very important activity, they are often not in the optimal organizational and functional conditions to develop an adequate "path of secondary prevention".

6. The problem of limited patient access to CPR is widely discussed in this position paper. This aspect is an element of great importance for healthcare planning. Beyond the obvious simplification, if in the United States it is believed that the increase in participation in CPR programs from $20 \%$ to $70 \%$ may lead to a reduction of 25,000 deaths and 180,000 hospitalizations per year 24 , it follows that every "plus or minus" percentage point of membership in national CPR programs equates to "an increase or decrease" of 500 deaths and 3600 hospitalizations per year. It is reasonable to suppose that public opinion, and perhaps even the system, is not very aware of these elements.

7. The need to give clear evidence of the results obtained from a CPR program in the individual patient, in a way that is easily understood by the institutional interlocutor and comparable with other rehabilitation disciplines. Continuing to use purely technical-cardiovascular language, referring to a set of parameters (e.g. NYHA class, peak V02, Watt, meters walked on the walk test, quality of life score, etc.), and not describing the outcome obtained in the person as a whole, has proven little effective in recent years.

\section{Lights}

1. The most important light is the effectiveness of CPR, demonstrated not only by the innumerable meta-analyses that have included mostly North American or Northern European cases and have been often cited in this position paper, but now also by large Italian national cases $[11,29]$, with results that are based on both clinical and administrative data and can be deduced from both the outpatient and inpatient content.

2. Multi-interdisciplinarity: this principle, which everyone now talks about and which has now become inflated in the medical conventions of each specialized branch, has been an integral part of CPR and its way of working for decades. The so-called chronic patient, often elderly and suffering from multiple pathologies, requires necessarily a multi-interdisciplinary treatment approach. The epidemiological reality places CPR in an ideal position to help provide an effective response to the current and future health needs of the population.

3. Its size. Despite the numerous "shadows" mentioned above, CPR is a reality of respectable dimensions. As reported in the last ISYDE.13-Directory [17], in 2013, there were 221 CPR facilities registered in our country, an increase compared to the previous 5year period, with more than 3200 beds and 102 outpatient sites. It is, hence, a non-negligible reality, serving both patients and the health system.

4. The cultural and scientific vivacity that has made CPR a real laboratory in which ideas and projects have been experimented and proposed to the scientific community, making the CPR community open to innovations on the one hand and challenges on the other.

\section{Prospects and needs}

1. CPR is called on to transmit the culture of prevention and rehabilitation with increasing effectiveness, decision and availability, outside its own confines. This is a mission that belongs 
to us because in CPR there is concentrated the maximum of professional competence and experience on this subject. No one has doubts about the fact that the know-how of hemodynamics, electrophysiology or coronary intensive care resides among professionals working in those fields. The same principle must also apply to CPR, and those who work in this sector must make themselves available to transmit our culture. For the good of patients, prevention and rehabilitation must start the moment a cardiac patient is admitted to hospital or visits the GP and not be seen as an "aftermath", i.e. pertaining always to someone else.

2. CPR must express its results, in the individual patient, through global indicators, applicable to the person in the various functional areas and based on scales, which at the same time are internationally recognized and understandable by the institutional interlocutors. For example, the identification and qualification of rehabilitation needs through the ICF (International Classification of Functioning) is possible and can represent an important topic of work and development in order to be able to express in the individual patient the outcomes of a CPR intervention in terms of functional recovery at the level of the person as a whole [88]. The epidemiological revolution we are currently witnessing will increasingly impose the adoption of functional outcomes centered on quality of life, both at the level of the individual patient and in clinical trials [30].

3. CPR must accept the need to measure itself in terms of its own results and declare minimum levels qualifying a CPR program. The experience of Minimal Care [20], developed by the non-medical areas of GICR-IACPR, should be an example to the entire CPR community. Declaring the minimum levels for a CPR program is necessary to discriminate between what is and what is not CPR according to evidence-based medicine.

4. CPR must fully accept the challenge imposed by epidemiology and the health system. Inpatient departments must be made increasingly available and be organized for complex patients, up to the point of developing UICRs. At the same time, it is necessary to develop and stimulate outpatient pathways for patients with moderate-low complexity, also to increase the rate of referral to CPR and so avoid that today's low-risk patients become high-risk patients tomorrow. Naturally, the above cannot be achieved in the absence of an adequate sensitivity to the issue on the part of the institutions.

5. Prevention is the key element to guarantee the sustainability of the health system in the near future. Secondary prevention, and therefore CPR, are destined to play a central role in this process. Of the importance of this the CPR community must become fully aware.

6. CPR must preside over the continuity of care path and move in the direction of "Individualized Medicine" and "Precision Medicine", entering into the e-Health and m-Health sectors. It is important that the anecdotal experiences of telemedicine surveyed by ISYDE.13-Directory [17] be developed and become care modes complementary to the traditional ones. It is important that the CPR community begins to use and becomes more and more familiar with terms such as biomarkers, genetics, and epigenetics.

7. The CPR sphere must connect ever more closely with that of the patient community. This is an important step to improve the awareness of their importance on the part of those who benefit from CPR programs, both at the local community level and at the Scientific Society level. In the absence of this connection it will be difficult to dispel the "shadows" mentioned above.

\section{References}

1. Piepoli MF, Hoes AW, Agewall S, et al. 2016 European Guidelines on cardiovascular disease prevention in clinical practice: The Sixth Joint Task Force of the European Society of Cardiology and Other Societies on Cardiovascular Disease Prevention in Clinical Practice (constituted by representatives of 10 societies and by invited experts). Developed with the special contribution of the European Association for Cardiovascular Prevention \& Rehabilitation (EACPR). Eur Heart J 2016;37:2315-81.

2. Piepoli MF, Corrà U, Adamopoulos $\mathrm{S}$, et al. Secondary prevention in the clinical management of patients with cardiovascular diseases. Core components, standards and outcome measures for referral and delivery: a policy statement from the cardiac rehabilitation section of the European Association for Cardiovascular Prevention \& Rehabilitation. Endorsed by the Committee for Practice Guidelines of the European Society of Cardiology. Eur J Prev Cardiol 2014;21:664-81.

3. Ibanez B, James S, Agewall S, et al. 2017 ESC Guidelines for the management of acute myocardial infarction in patients presenting with ST-segment elevation: The Task Force for the management of acute myocardial infarction in patients presenting with ST-segment elevation of the European Society of Cardiology (ESC). Eur Heart J 2018;39:119-77.

4. Aguero F, Marrugat J, Elosua R, et al.; REGICOR Investigators. New myocardial infarction definition affects incidence, mortality, hospitalization rates and prognosis. Eur J Prev Cardiol 2015;22:1272-80.

5. Montalescot G, Sechtem U, Achenbach S, et al. 2013 ESC guidelines on the management of stable coronary artery disease: The Task Force on the management of stable coronary artery disease of the European Society of Cardiology. Eur Heart J 2013;34:2949-3003.

6. Ponikowski P, Voors AA, Anker SD, et al. 2016 ESC Guidelines for the diagnosis and treatment of acute and chronic heart failure: The Task Force for the diagnosis and treatment of acute and chronic heart failure of the European Society of Cardiology (ESC). Developed with the special contribution of the Heart Failure Association (HFA) of the ESC. Eur Heart J 2016;37:2129-200.

7. Greco C, Rosato S, D’Errigo P, Mureddu GF, Lacorte E, Seccareccia F. Trends in mortality and heart failure after acute myocardial infarction in Italy from 2001 to 2011. Int J Cardiol 2015;184:115-21.

8. Mureddu GF, D’Errigo P, Rosato S, Seccareccia F, Greco C. The hazard of major adverse cardiac events in high thrombotic risk patients is stable until 5 years after an acute myocardial infarction [abstract]. Eur Heart J 2017;38(Suppl 1):515.

9. Griffo R, Ambrosetti M, Tramarin R, et al.; ICAROS Investigators. Effective secondary prevention through cardiac rehabilitation after coronary revascularization and predictors of poor adherence to lifestyle modification and medication. Results of the ICAROS Survey. Int J Cardiol 2013;167:1390-5.

10. Giannuzzi P, Temporelli PL, Marchioli R, et al.; GOSPEL Investigators. Global secondary prevention strategies to limit event recurrence after myocardial infarction: results of the GOSPEL study, a multicenter, randomized controlled trial from the Italian Cardiac Rehabilitation Network. Arch Intern Med 2008;168:2194-204.

11. Doimo S, Fabris E, Piepoli M et al. Impact of ambulatory cardiac rehabilitation on cardiovascular outcomes: a long-term follow-up study. Eur Heart J 2018. doi: 10.1093/eurheartj/ehy417 [Epub ahead of print].

12. Rauch B, Davos CH, Doherty P, et al. The prognostic effect of cardiac rehabilitation in the era of acute revascularization and statin therapy: a systematic review and meta-analysis of 
randomized and non-randomized studies - The Cardiac Rehabilitation Outcome Study (CROS). Eur J Prev Cardiol 2016;23:1914-39.

13. Anderson L, Oldridge N, Thompson DR, et al. Exercise-based cardiac rehabilitation for coronary heart disease: Cochrane systematic review and meta-analysis. J Am Coll Cardiol 2016;67:1-12.

14. Taylor RS, Sagar VA, Davies EJ, et al. Exercise-based rehabilitation for heart failure. Cochrane Database Syst Rev 2014;4:CD003331.

15. Butchart EG, Gohlke-Barwolf C, Antunes MJ, et al.; Working Groups on Valvular Heart Disease, Thrombosis, and Cardiac Rehabilitation and Exercise Physiology, European Society of Cardiology. Recommendations for the management of patients after heart valve surgery. Eur Heart J 2005;26:2463-71.

16. Sandesara PB, Lambert CT, Gordon NF, et al. Cardiac rehabilitation and risk reduction: time to "rebrand and reinvigorate". J Am Coll Cardiol 2015;65:389-95.

17. Griffo R, Tramarin R, Volterrani M, et al. Italian Survey on Cardiac Rehabilitation (ISYDE.13-Directory): report su strutture, organizzazione e programmi di cardiologia riabilitativa in Italia. G Ital Cardiol 2016;17:217-24.

18. Pesah E, Supervia M, Turk-Adawi K, Grace SL. A review of cardiac rehabilitation delivery around the world. Prog Cardiovasc Dis 2017;60:267-80.

19. Pastormerlo LE, Aimo A, Piepoli M, Emdin M. The bottleneck of cardiac rehabilitation for patients with coronary artery disease: how to overcome Eur J Prev Cardiol 2018;25:1239-41.

20. Fattirolli F, Bettinardi 0, Angelino E, et al. What constitutes the "minimal care" interventions of the nurse, physiotherapist, dietician and psychologist in cardiovascular rehabilitation and secondary prevention: a position paper from the Italian Association for Cardiovascular Prevention, Rehabilitation and Epidemiology. Eur J Prev Cardiol 2018 Aug 1. doi: 10.1177/2047487318789497 [Epub ahead of print].

21. Kwan G, Balady GJ. Cardiac rehabilitation 2012: advancing the field through emerging science. Circulation 2012;125:e369-73.

22. Secondary cardiovascular disease prevention and control. A World Heart Federation report. July 2014. Accessed on: 8 October 2018. Available from: ttp://ss1.spletnik.si/4_4/000/000/41d/f04/ 2ndary-Prevention-Report.pdf.

23. Bjarnason-Wehrens B, McGee H, Zwisler AD, et al. Cardiac rehabilitation in Europe: results from the European Cardiac Rehabilitation Inventory Survey. Cardiac Rehabilitation Section European Association of Cardiovascular Prevention and Rehabilitation. Eur J Cardiovasc Prev Rehabil 2010;17:410-8.

24. Lobelo F, Rohm Young D, Sallis R, et al. Routine assessment and promotion of physical activity in healthcare settings. a scientific statement from the American Heart Association. Circulation 2018;137:e495-522.

25. Randal JT, Balady G, Banka G, et al. 2018 ACC/AHA clinical performance and quality measures for cardiac rehabilitation: a report of the American College of Cardiology/American Heart Association Task Force on Performance Measures. J Am Coll Cardiol 2018;71:1814-37.

26. Greco C, Cacciatore G, Gulizia M, et al. Associazione Nazionale Medici Cardiologi Ospedalieri; Italian Association for Cardiovascular Prevention, Rehabilitation and Epidemiology; Gruppo Italiano di Cardiologia Riabilitativa. Criteri per la selezione dei pazienti da inviare ai centri di cardiologia riabilitativa. Monaldi Arch Chest Dis 2011;76:1-12.

27. Greco C, Bovenzi FM, Berti S, et al. Documento ANMCO/GICRIACPR/GISE: L'organizzazione dell'assistenza nella fase post-acuta delle sindromi coronariche. G Ital Cardiol 2014;15(1 Suppl 1):3S-27S.
28. 0'Connor CM, Whellan DJ, Lee KL, et al.; HF-ACTION Investigators. Efficacy and safety of exercise training in patients with chronic heart failure: HF-ACTION randomized controlled trial. JAMA 2009;301:1439-50.

29. Scalvini S, Grossetti F, Paganoni AM, La Rovere MT, Pedretti R, Frigerio M; HF Data Project. Cardiac rehabilitation referral in Lombardy Region: a population study on incident cases from 2005 to 2012 [abstract]. Eur Heart J 2018;39(Suppl 1):566.

30. Bell SP, Orr NM, Dodson JA, et al. What to expect from the evolving field of geriatric cardiology. J Am Coll Cardiol 2015;66:1286-99.

31. Forman DE, Maurer MS, Boyd C, et al. Multimorbidity in older adults with cardiovascular disease. J Am Coll Cardiol 2018;71:2149-61.

32. Vigorito C, Abreu A, Ambrosetti M, et al. Frailty and cardiac rehabilitation: a call to action from the EAPC Cardiac Rehabilitation Section. Eur J Prev Cardiol 2017;24:577-90.

33. Afilalo J, Lauck S, Kim DH, et al. Frailty in older adults undergoing aortic valve replacement: The FRAILTY-AVR Study. J Am Coll Cardiol 2017;70:689-700.

34. Reeves GR, Whellan DJ, 0'Connor CM, et al. A novel rehabilitation intervention for older patients with acute decompensated heart failure: The REHAB-HF Pilot Study. JACC Heart Fail 2017;5:359-66.

35. Di Tano G, De Maria R, Gonzini L, et al. IN-HF Outcome Investigators. The 30-day metric in acute heart failure revisited: data from IN-HF Outcome, an Italian nationwide cardiology registry. Eur J Heart Fail 2015;17:1032-41.

36. Bachmann JM, Duncan MS, Shah AS, et al. Association of cardiac rehabilitation with decreased hospitalizations and mortality after ventricular assist device implantation. JACC Heart Fail 2018;6:130-9.

37. Corrà U, Pistono M, Mezzani A, et al. Cardiovascular prevention and rehabilitation for patients with ventricular assist device from exercise therapy to long-term therapy. Part I: Exercise therapy. Monaldi Arch Chest Dis 2011;76:27-32.

38. Fang JC, Ewald GA, Allen LA, et al. Advanced (stage D) heart failure: a statement from the Heart Failure Society of America Guidelines Committee. J Card Fail 2015;21:519-34.

39. Ministero della Salute. [Piano di indirizzo per la Riabilitazione del Ministero della Salute del 2011].[Document in Italian]. Accessed on: 8 October 2018. Available from: http://www.salute.gov.it/imgs/ C_17_pubblicazioni_1546_allegato.pdf

40. Griffo R, Ambrosetti M, Furgi G, et al. [Gruppo Italiano di Cardiologia Riabilitativa e Preventiva. Requisiti e indicatori in cardiologia riabilitativa: documento di consenso del GICR/IACPR].[Article in Italian]. Monaldi Arch Chest Dis 2012;78:166-92.

41. Greco C, Cacciatore G, Gulizia M, et al. [Criteri per la selezione dei pazienti da inviare ai centri di cardiologia riabilitativa].[Article in Italian ]. G Ital Cardiol 2011;12:219-29.

42. Anderson L, Sharp GA, Norton JR, et al. Home-based versus centre-based cardiac rehabilitation. Cochrane Database Syst Rev 2017;6:CD007130.

43. Frederix I, Vanhees L, Dendale P, Goetschckx K. A review of telerehabilitation for cardiac patients. J Telemed Telecare 2015;21:45-53.

44. Davis MS. Variations in patients' compliance with doctors' advice: an empirical analysis of patterns of communication. Am J Public Health 1968;58:274-88.

45. World Health Organization. Adherence to long-term therapies: evidence for action. Geneva: WHO; 2003.

46. Majani G. [Compliance, adesione, aderenza. I punti critici della relazione terapeutica].[Book in Italian]. Milano: McGraw-Hill; 2001.

47. Vrijens B, De Geest S, Hughes DA, et al. ABC Project Team. A new taxonomy for describing and defining adherence to medications. Br J Clin Pharmacol 2012;73:691-705. 
48. Brown MT, Bussell JK. Medication adherence: WHO cares? Mayo Clin Proc 2011;86:304-14.

49. Elliott RA. Problems with medication use in the elderly: an Australian perspective. Geriatric Therapeutics 2006;36:58-66.

50. Agenzia Italiana del Farmaco. [L'uso dei farmaci in Italia. Rapporto Nazionale gennaio-settembre 2016. Gennaio 2017].[Report in Italian]. Accessed on: 8 October 2018. Available from: http://www.aifa.gov.it/sites/default/files/Rapporto_OsMed_gennaio _settembre_2016.pdf

51. Steiner JF, Earnest MA. The language of medication-taking. Ann Intern Med 2000;132:926-30.

52. Caldeira D, Vaz-Carneiro A, Costa J. The impact of dosing frequency on medication adherence in chronic cardiovascular disease: systematic review and meta-analysis. Rev Port Cardiol 2014;33:431-7.

53. Rambhade S, Chakarborty A, Shrivastava A, Patil UK, Rambhade A. A survey on polypharmacy and use of inappropriate medications. Toxicol Int 2012;19:68-73.

54. Naderi S, Bestwick J, Wald D. Adherence to drugs that prevent cardiovascular disease: meta-analysis on 376,162 patients. Am J Med 2012;125:882-7.

55. Osterberg L, Blaschke T. Adherence to medication. N Engl J Med 2005;353:487-97.

56. Senst BL, Achusim LE, Genest RP, et al. Practical approach to determining costs and frequency of adverse drug events in a health care network. Am J Health Syst Pharm 2001;58:1126-32.

57. Bansilal S, CastellanoJ, Garrido E, et al. Assessing the impact of medication adherence on long-term cardiovascular outcomes. J Am Coll Cardiol 2016;68:789-801.

58. Riles EM, Jain AV, Fendrick AM. Medication adherence and heart failure. Curr Cardiol Rep 2014;16:458.

59. Miró 0, Estruch R, Martín-Sánchez FJ, et al. ICA-SEMES Research Group. Adherence to Mediterranean diet and all-cause mortality after an episode of acute heart failure: results of the MEDIT-AHF Study. JACC Heart Fail 2018;6:52-62.

60. Flora PK, McMahon CJ, Locke SR, Brawley LR. Perceiving cardiac rehabilitation staff as mainly responsible for exercise: a dilemma for future self-management. Appl Psychol Health Well Being 2018;10:108-26.

61. American Geriatrics Society Expert Panel on the Care of Older Adults with Multimorbidity. Patient-centered care for older adults with multiple chronic conditions: a stepwise approach from the American Geriatrics Society: American Geriatrics Society Expert Panel on the Care of Older Adults with Multimorbidity. J Am Geriatr Soc 2012;60:1957-68.

62. Menezes AR, Lavie CJ, Forman DE, et al. Cardiac rehabilitation in the elderly. Prog Cardiovasc Dis 2014;57:152-9.

63. Rich MW, Chyun DA, Skolnick AH, et al.; American Heart Association Older Populations Committee of the Council on Clinical Cardiology, Council on Cardiovascular and Stroke Nursing, Council on Cardiovascular Surgery and Anesthesia, and Stroke Council; American College of Cardiology; and American Geriatrics Society. Knowledge gaps in cardiovascular care of the older adult population: a scientific statement from the American Heart Association, American College of Cardiology, and American Geriatrics Society. J Am Coll Cardiol 2016;67:2419-40.

64. Vigorito C, Giallauria F. Effects of exercise on cardiovascular performance in the elderly. Front Physiol 2014;5:51.

65. Forman DE, Arena R, Boxer R, et al.; American Heart Association Council on Clinical Cardiology; Council on Cardiovascular and Stroke Nursing; Council on Quality of Care and Outcomes Research; and Stroke Council. Prioritizing functional capacity as a principal end point for therapies oriented to older adults with cardiovascular disease: a scientific statement for healthcare professionals from the American Heart Association. Circulation 2017;135:e894-918.

66. Gillebert TC, Brooks N, Fontes-Carvalho R, et al. ESC core curriculum for the general cardiologist (2013). Eur Heart J 2013;34:2381-411.

67. Flaherty G, Abdul Shukur AS, Daly J, Jones J. Proposal for a standardised inter-professional undergraduate curriculum in preventive cardiology. Eur J Prev Cardiol 2016;23:86-7.

68. Istituto Nazionale di Statistica. [Le organizzazioni di volontariato in Italia. Anno 2003]. [Document in Italian]. Accessed on: 8 October 2018. Available from: https//ebiblio.istat.it/digibib/Organizzazioni\%20Volontariato/ IST0034663Le_organizzazioni_di_volontariato_in_Italia_2003.pdf

69. Linee guida del Ministero della Sanità per le attività di riabilitazione. Gazzetta Ufficiale, 30 maggio 1998, n. 124.

70. Regione Lombardia. [Decreto Direzione Generale Sanità n. 6910 del 26/06/2008. Appropriatezza dei ricoveri riabilitativi in rapporto all'attuale offerta di degenza in Regione Lombardia].[Regional law in Italian].

71. Regione Lombardia. [Deliberazione n. 19883 del 16 dicembre 2004. Riordino della rete delle attività di riabilitazione].[Regional law in Italian]. Accessed on: 8 October 2018. Available from: http:/www.terzaeta.com/database/leggi-e-decreti-regionali//ombardia/ burl2004_53_dgr19883.pdf

72. Linee guida per la compilazione e la codifica ICD-9-CM della Scheda di Dimissione Ospedaliera. Deliberazione Generale della Regione Veneto n. 1169 del 19 luglio 2016.

73. Regione Sicilia. [Il Piano della riabilitazione].[Document in Italian]. Official Journal of the Region Sicily, 21 December 2012, vol. 66, n. 54.

74. Ministero della Salute. [Metodologia per la definizione dei criteri/ parametri di appropriatezza ed efficienza dei ricoveri di riabilitazione ospedaliera. Report risultati 2010-2012]. [Report in Italian]. Accessed on: 8 October 2018. Available from: http:/www.salute.gov.it/portale/ documentazione/p6_2_2_1.jsp?lingua=italiano\&id=2159

75. Chan PS, Oetgen WJ, Buchanan D, et al. Cardiac performance measure compliance in outpatients: the American College of Cardiology and National Cardiovascular Data Registry's Pinnacle (Practice Innovation and Clinical Excellence) Program. J Am Coll Cardiol 2010;56:8-14.

76. Resurrección DM, Moreno-Peral P, Gómez-Herranz M, et al. Factors associated with non-participation in and dropout from cardiac rehabilitation programmes: a systematic review of prospective cohort studies. Eur J Cardiovasc Nurs 2018. doi: 10.1177/1474515118783157 [Epub ahead of print].

77. Ministero della Salute. La centralità della persona in riabilitazione: nuovi modelli organizzativi e gestionali. Quaderni del Ministero della Salute n. 8, marzo-aprile 2011.

78. Bayes-Genis A, Voors AA, Zannad F, James L. Januzzi JL, Richards AM. Transitioning from usual care to biomarker based personalized and precision medicine in heart failure: call for action. Eur Heart $\mathrm{J}$ 2018;39:2793-8.

79. Bäck M, Hansen TB, Frederix I. Cardiac rehabilitation and exercise training recommendations. Cardiac rehabilitation: rationale, indications and core components. 30 Jun 2017. Accessed on: 8 October 2018. Available from: https://www.escardio.org/ Education/ESC-Prevention-of-CVD-Programme/Rehabilitation

80. Maddox TM, Albert NM, Borden WB, et al. The learning healthcare system and cardiovascular care: a scientific statement from the American Heart Association. Circulation 2017;135:e826-57.

81. Bhatt DL, Drozda JP Jr, Shahian DM, et al. ACC/AHA/STS statement on the future of registries and the performance measurement enterprise: a report of the American College of Cardiology/ American Heart Association Performance Measures and the Society of Thoracic Surgeons. J Am Coll Cardiol 2015;66:2230-45. 
82. Windle JR, Katz AS, Dow JP Jr, et al. ACC/ASE/ASNC/HRS/SCAI health policy statement on integrating the health care enterprises. J Am Coll Cardiol 2016;68:1348-64.

83. Goodman SN, Schneeweiss S, Baiocchi M. Using design thinking to differentiate useful from misleading evidence in observational research. JAMA 2017;317:705-7.

84. Bhavnani SP, Parakh K, Atreja A, et al. 2017 Roadmap for innovation-ACC health policy statement on healthcare transformation in the era of digital health, big data, and precision health: a report of the American College of Cardiology Task Force on Health Policy Statements and Systems of Care. J Am Coll Cardiol 2017;70:2697-718.

85. Psaty BM, Dekkers OM, Cooper RS. Comparison of 2 treatment models: precision medicine and preventive medicine. JAMA 2018;320:751-2.

86. Ades PA, Keteyian SJ, Wright JS, et al. Increasing cardiac rehabilitation participation from $20 \%$ to $70 \%$ : a road map from the Million Hearts Cardiac Rehabilitation Collaborative. Mayo Clin Proc 2017;92:234-42.

87. West RR, Jones DA, Henderson AH. Rehabilitation after myocardial infarction trial (RAMIT): multi-centre randomised controlled trial of comprehensive cardiac rehabilitation in patients following acute myocardial infarction. Heart 2012;98:637-44.

88. Giardini A, Traversoni S, Garbelli C, Lodigiani A. Digitalisation and clinical care pathways in rehabilitation medicine: a possible integration from the goal-planning and the rehabilitation programme design to the evaluation of clinical outcomes. G Ital Med Lav Ergon 2018;40:22-9.

89. Ambrosetti M, Doherty P, Faggiano P, et al. Characteristics of structured physical training currently provided in cardiac patients: insights from the Exercise Training in Cardiac Rehabilitation (ETCR) Italian survey. Monaldi Arch Chest Dis 2017;87:778.

\section{Reviewers of the document}

Daniele Bertoli (S.C. Cardiologia Clinica Riabilitativa, ASL Spezzino, La Spezia, Italy), Andrea Bianco (S.S.D. Cardioriabilitazione e Valutazione Funzionale, A.0. Brotzu, Cagliari, Italy), Pasqualina Calisi (S.S.D. Cardiologia Riabilitativa, Ospedale La Colletta, Arenzano, Italy), Carlo Ciglia (U.O. Cardiologia, Villa Pini d'Abruzzo, Chieti, Italy), Furio Colivicchi (U.O.C. Cardiologia, A.C.O. San Filippo Neri, Roma, Italy), Anna Frisinghelli (U.0. Cardiologia Riabilitativa, Presidio Ospedaliero di Passirana, ASST Rhodense, Garbagnate M.se, Italy), Michele Gabriele (U.0.C. Cardiologia, Ospedale Abele Ajello, Mazara del Vallo, Italy), Giuseppe Giancamerla (Conacuore, Aosta, Italy), Rocco Lagioia (U.0. Cardiologia Riabilitativa, Dipartimento di Cardioangiologia Riabilitativa, Istituti Clinici Scientifici Maugeri IRCCS di Cassano delle Murge, Italy), Roberto Marini (Ambulatorio Policlinico Salus, Trieste, Italy), Bruna Miserrafiti (U.0. Riabilitazione Cardio-Respiratoria, Presidio Ospedaliero Tiberio Evoli, Melito Porto Salvo, Italy), Salvatore Pirelli (Past-President GICR-IACPR, Past-President ANMCO, Milano, Italy), Matteo Ruzzolini (U.0. Cardiologia, Ospedale Fatebenefratelli, Roma, Italy), Gianpaolo Scorcu (S.S.D. di Valutazione, Diagnosi e Consulenza Cardiologica, A.0. Brotzu, Cagliari, Italy), Franco Tarro Genta (U.0. Cardiologia Riabilitativa, Istituti Clinici Scientifici Maugeri, Presidio Sanitario di Torino, Italy), Nidal Tourkmani (Preventive Cardiology and Cariac rehabilitation ABL, Swiss Prestige International Medical Centre-Guangzhou, China), Elio Venturini (U.0. Cardiologia e Riabilitazione Cardiologica, Ospedale Civile, Cecina, Italy), Marika Werren (S.O.S. Cardiologia Riabilitativa, Istituto di Medicina Fisica e Riabilitazione Gervasutta, Udine, Italy), Gianni Zobbi (U.0. Cardiologia, Castelnovo Monti, A.0. di Reggio Emilia, Italy). 